\title{
1 Palm-to-finger cortical functional interactions in primary 2 somatosensory cortex: a 7T fMRI study
}

3

4 Michel Akselrod ${ }^{1,2,3}$, Roberto Martuzzi ${ }^{4,2}$, Wietske van der Zwaag ${ }^{5}$, Olaf Blanke ${ }^{2,6,+}$ and Andrea Serino $0^{1,2,+}$

6

${ }^{1}$ Laboratory MySpace, Department of Clinical Neuroscience, University Hospital of Lausanne (CHUV), Switzerland

${ }^{2}$ Laboratory of Cognitive Neuroscience, Brain Mind Institute and Center for Neuroprosthetics, Swiss Federal Institute

9 of Technology of Lausanne (EPFL), Campus Biotech, Geneva, Switzerland

$10{ }^{3}$ Minded Program, CMON unit, Italian Institute of Technology, Genova, Italy

11 'Foundation Campus Biotech Geneva, Geneva, Switzerland

12 Spinoza Centre for Neuroimaging, Amsterdam, Netherlands

$13{ }^{6}$ Department of Neurology, University Hospital, Geneva, Switzerland

14 +equal contributions

17 Corresponding authors:

18 Michel Akselrod

Olaf Blanke

19 Laboratory MySpace

Laboratory of Cognitive Neuroscience

20 Department of Clinical Neuroscience

Center for Neuroprosthetics

21 University Hospital of Lausanne (CHUV) Ecole Polytechnique Fédérale de Lausanne (EPFL)

22 Hospital Nestlé

23 Rue Pierre-Decker 4

$24 \mathrm{CH}-1011$ Lausanne, Switzerland

25 michel.akselrod@gmail.com
Campus Biotech $\mathrm{H} 4$

Chemin des Mines 9

$\mathrm{CH}-1202$ Geneva, Switzerland

olaf.blanke@epfl.ch 


\section{ACKNOWLEDGEMENTS}

\section{2 funding statement}

3 This work was supported by the Bertarelli Foundation and the National Competence

4 Centre for Biomedical Imaging (NCCBI, Switzerland, grant number 591108). MA is

5 supported by the European Union's Horizon 2020 research and innovation programme

6 under the Marie Skłodowska-Curie grant agreement No 754490 - MINDED project.

7 AS is supported by the Swiss National Science Foundation (PP00P3_163951/1).

8

9 conflict of interest disclosure

10 None of the authors has competing interests.

11

\section{2 ethics approval statement}

13 Research was conducted under the approval of the Ethics Committee of the University

14 of Lausanne and written informed consent was obtained from all experimental 15 subjects.

16 


\section{$1 \quad$ ABSTRACT}

2 Many studies focused on the cortical representations of fingers, while the palm is

3 relatively neglected despite its importance for hand function. Here, we investigated 4 palm representation (PR) and its interactions with finger representations (FRs) in

5 primary somatosensory cortex (S1). Few studies in humans suggested that PR is

6 located medially with respect to FRs in S1, yet to date, no study directly quantified the

7 somatotopic organization of PR and the five FRs. Importantly, the relationship between

8 the somatotopic organization and the cortical functional interactions between PR and

9 FRs remains largely unexplored. Using 7T fMRI, we mapped PR and the five FRs at 10 the single subject level. First, we analyzed the cortical distance between PR and FRs

11 to determine their somatotopic organization. Results show that the PR was located 12 medially with respect to D5. Second, we tested whether the observed cortical 13 distances would predict palm-finger functional interactions. Using three 14 complementary measures of functional interactions (co-activations, pattern similarity 15 and resting-state connectivity), we show that palm-finger functional interactions were 16 not determined by their somatotopic organization, that is, there was no gradient moving 17 from D5 to D1, except for resting-state connectivity, which was predicted by the 18 somatotopy. Instead, we show that the representational geometry of palm-finger 19 functional interactions reflected the physical structure of the hand. Collectively, our 20 findings suggest that the spatial proximity between topographically organized neuronal 21 populations do not necessarily predicts their functional interactions, rather the structure 22 of the sensory space (e.g. the hand shape) better predicts the observed functional 23 interactions. 


\section{1. INTRODUCTION}

2 The sensory cortices of the mammalian brain are topographically organized to form structured maps of the represented sensory features. This organizational principle is preserved across species, sensory modalities and individuals (Kaas, 1997; Uddin and Fawcett, 1988) and the role of this topographic organization and its relevance for functional brain interactions are important topics for fundamental and clinical research. Due to metabolic and structural constraints, the spatial proximity between topographically organized neuronal populations directly impacts their functional interactions (i.e. spatially close neurons are more likely to form synapses than distant ones) (van Ooyen et al., 2014; van Pelt et al., 2013). On the other hand, the statistics

11 of natural stimulation received during everyday life drives the tuning of functional 12 neuronal interactions through activity-dependent plasticity and can reinforce functional 13 interactions between distant neuronal populations (Buonomano and Merzenich, 14 1998). The somatosensory system is a particularly relevant model to study the relationship between topographic organization in neural maps, functional interactions and use-related function. The somatosensory system must support the functional interactions between elements (i.e. body parts) that can move with respect to each other (e.g. the configuration of the five fingers during object manipulation), can directly interact with each other (i.e. self-touch) and need to fulfill many different sensorimotor functions. While the somatosensory representations of fingers have been researched extensively, the palm has been less studied despite its importance for hand function and despite being anatomically connected with all five fingers. The aim of the present study is to investigate the topographical and functional organization of tactile representations of the palm (palm representation, PR) and its functional interactions with the tactile representations of the five fingers (fingers representations, FRs) in the human primary somatosensory cortex, $\mathrm{S} 1$.

$\mathrm{S} 1$ is somatotopically organized into a cortical map of the contralateral half of the body (Kaas et al., 1979, Rasmussen and Penfield, 1947). FRs in S1 appear in a lateromedial sequence (D1 - D2 - D3 - D4 - D5). This organization is consistent across individuals as shown in recent ultra-high field (7T) fMRI studies (Besle et al., 2014; Martuzzi et al. 2014; Sanchez-Panchuelo et al. 2010, 2012, 2014; Schweisfurth et al., 2011, 2014, 2018; Schweizer et al., 2008; Stringer et al. 2011, 2014). While the $\mathrm{S} 1$ representations of the base of the fingers (i.e. the distal part of the palm) with respect to FRs have been described in a consistent manner (Blankenburg et al., 2003; Sanchez-Panchuelo et al. 2012, 2014; Schweisfurth et al., 2011, 2014), reports concerning the $\mathrm{S} 1$ representations of the proximal part of the palm, which is 
1 the focus of the present study, are mixed. Several studies suggested that PR is located

2 medially with respect to FRs in S1 (Blankenburg et al., 2003; Moore et al., 2000;

3 Rasmussen and Penfield, 1947). However, other studies, specifically aiming at 4 mapping FRs and PR, failed to detect a clear palm representation and could not determine the palm to finger sequence in human S1, likely because they used neuroimaging without sufficient spatial resolution (MEG) or tactile stimulation protocols non-optimized for mapping PR (Hashimoto et al., 1999; Sanchez-Panchuelo et al., 2012). Importantly, none of these studies provided direct quantification of the somatotopic organization of the 5 fingers and the palm.

11 The present study aimed at mapping bilateral PR and FRs in S1 at the level of single 12 subjects and investigate their somatotopic organization and cortical functional 13 interactions. To this aim, we applied a 7T fMRI mapping procedure that was validated 14 in previous studies (Akselrod et al., 2017; Martuzzi et al., 2014, 2015; Mehring et al., 2019; Serino et al., 2017). First, we analyzed the cortical distance between PR and the five FRs to determine the somatotopic sequence in S1. This was done in order to validate the proposed serial somatotopic arrangement D1 - D2 - D3 - D4 - D5 PALM. Second, in order to evaluate the relationship between somatotopic and functional organizations, we tested to which extent the functional interactions between $\mathrm{PR}$ and FRs matched their somatotopic organization. We performed the following analyses to obtain complementary measures of functional interactions between PR and FRs: 1) we measured the degree of co-activation between PR and FRs during tactile stimulation of the palm and of the fingers; 2) we compared the multi-voxel patterns of activity in $\mathrm{S} 1$ during tactile stimulation of the palm and of the fingers; 3 ) we quantified the resting-state functional connectivity between PR and FRs.

Based on previous reports in humans (Blankenburg et al., 2003; Moore et al., 2000;

Rasmussen and Penfield, 1947), we predicted that PR would be located medially with respect to D5 corresponding to a serial somatotopic arrangement. It is possible that the somatotopic arrangement of PR and FRs affects their functional interactions (i.e. stronger functional interactions between closer representations); if this was the case, a serial somatotopic arrangement would predict that functional interactions should be strongest between PALM-D5 representations, with a further decreasing gradient from D4 to D1 and the weakest interaction between PALM-D1

35 representations. However, considering that the palm is often recruited concomitantly 36 with the five fingers during most in-hand manipulation activities (Bullock and Dollar, 37 2011; Pont et al., 2009) and that functional interactions between hand representations 
1 reflect the natural usage of hands (Ejaz et al., 2015), we hypothesize that functional

2 interactions between PR and FRs should not match a potential serial somatotopic

3 arrangement (i.e. the palm would not interact preferentially with D5, then D4, D3, ...).

4 To provide further insights into the relationship between hand somatotopic

5 organization and hand functional interactions, we compared the representational

6 geometry of the aforementioned measures (i.e. cortical distances, co-activations,

7 multi-voxel activity patterns and functional connectivity) with various competitive

8 models: two models based on the physical structure of the hand ("Body model" and

9 "Perceived body model") and two purely conceptual models based on different 10 possible configurations of palm and fingers ("Linear model" and "Circular model").

\section{2. METHODS}

\section{$13 \quad 2.1$ Subjects}

1415 healthy subjects ( 5 females) aged between 18 and 39 years old (mean \pm std: 24.3 $15 \pm 5.2$ years) participated in the study. One participant was excluded due to excessive 16 head motion during MRI acquisition (up to $5 \mathrm{~mm}$ of movement in the z-direction).

17 Data from another group of 9 healthy subjects ( 5 females, aged between 26 and 33 18 years old) recruited in a previous study (Mehring et al., 2019) was used to extract 19 average hand dimensions (see section 2.11).

20 All participants were right-handed as assessed orally using the Edinburgh Handedness 21 Inventory (Oldfield, 1971).

22 All subjects gave written informed consent, all procedures were approved by the Ethics 23 Committee of the Faculty of Biology and Medicine of the University of Lausanne, and 24 the study was conducted in accordance with the Declaration of Helsinki.

\subsection{Experimental procedure}

During $\mathrm{fMRI}$ acquisition, subjects received tactile stimulation on six skin regions on both hands (D1 - D2 - D3 - D4 - D5 - PALM). Tactile stimulation consisted of a gentle manual stroking at a rate of approximately $1 \mathrm{~Hz}$ performed by an experimenter with his index finger, who received instructions by means of MR compatible earphones. To reduce the variability of the tactile stimulation across participants and to guarantee that a reliable and constant pressure was exerted, the stroking was always performed by the same experimenter, who received extensive training prior to data acquisition. As shown in previous studies, natural touch induces very reliable BOLD signal responses in S1 and is well suited to study body representations in S1 (Akselrod et al., 2017; 
1 participant's fingers were repeatedly stroked on the two distal phalanges (thus

2 preventing contamination with palm stimulation), and the palm was stroked in the

3 center on a portion of skin of comparable size. One fMRI run for each hand was

4 acquired in pseudo-randomized order across participants. Within each run, the six regions of the same hand were stroked in a fixed order (D1 - D3 - D5 - D2 - D4 - PALM) and the sequence was repeated 4 times. Stimulation periods of $20 \mathrm{~s}$ were interleaved with periods of $10 \mathrm{~s}$ of rest (rest periods with no tactile stimulation). In addition to tactile stimulation runs, resting-state data ( $5 \mathrm{~min}$, eyes closed) and anatomical images were acquired for each participant.

\subsection{Data acquisition}

12 MR images were acquired using a short-bore head-only 7 Tesla scanner (Siemens 13 Medical, Germany) equipped with a 32-channel Tx/Rx RF-coil (Nova Medical, USA) 14 (Salomon et al., 2014). Functional images were acquired using a sinusoidal readout 15 EPI sequence (Speck et al., 2008) and comprised of 28 axial slices placed approximately orthogonal to the postcentral gyrus (voxel resolution $=1.3 \times 1.3 \times 1.3 \mathrm{~mm}^{3}$, $\mathrm{TR}=2 \mathrm{~s}, \mathrm{TE}=27 \mathrm{~ms}$, flip angle $=75^{\circ}$, matrix size $=160 \times 160, \mathrm{FOV}=210 \mathrm{~mm}$, GRAPPA factor=2). The mapping sequence included 361 volumes for each run and the restingstate sequence included 150 volumes. For the resting-state sequence, cardiac and respiratory signals were acquired. Anatomical images were acquired using an MP2RAGE sequence (Marques et al., 2010, resolution $=1 \times 1 \times 1 \mathrm{~mm}^{3}, \mathrm{TE}=2.63 \mathrm{~ms}$, TR $=7.2 \mathrm{~ms}, \mathrm{Tl} 1=0.9 \mathrm{sec}, \mathrm{TI} 2=3.2 \mathrm{sec}, \mathrm{TRmprage}=5 \mathrm{sec}$ ). To aid coregistration between the functional and the anatomical images, a whole brain EPI volume was also acquired with the same inclination used in the functional runs ( 81 slices, resolution $=1.3 \times 1.3 \times 1.3$ $\mathrm{mm}^{3}, \mathrm{TR}=5 \mathrm{~s}, \mathrm{TE}=27 \mathrm{~ms}$, flip angle $=75^{\circ}$, matrix size $=160 \times 160, \mathrm{FOV}=210 \mathrm{~mm}, \mathrm{GRAPPA}$ factor=2).

\subsection{Data preprocessing}

All images were preprocessed using the SPM8 software (Wellcome Department of

30 Cognitive Neurology, London, UK). Preprocessing of fMRI data included slice timing 31 correction, spatial realignment, and minimal smoothing (FWHM=2mm). Freesurfer 32 (http://surfer.nmr.mgh.harvard.edu/, version 6) was used for surface reconstruction 33 (recon-all), for computing cortical distance along the surface (see section 2.6) and for 34 surface rendering of $S 1$ hand maps of a representative subject (Fig.1C). The MRIcron software was used for visualizing results in 3D space for all subjects (McCausland

36 Center for Brain Imaging, University of South Carolina, US, 37 http://www.mccauslandcenter.sc.edu/mricro/mricron). The Connectome Mapper 3 
1 software was used for anatomical parcellation of each subject's mp2rage data in native

2 space (Tourbier et al. 2020).

\subsection{Definition of somatosensory hand representations}

Independently for each subject and each hand, the clusters corresponding to the representations of each stimulated hand region were delimited using an automated approach validated in previous publications (Akselrod et al., 2017; Martuzzi et al., 2014, 2015; Serino et al., 2017). A GLM analysis (SPM8) was carried out to estimate the response induced by the stimulation of the different hand regions. The model included 6 regressors (one for each stimulated hand region) convolved with the

11 hemodynamic response and with the corresponding first-order time derivatives, as well

12 as the 6 rigid-body motion parameters as nuisance regressors. For each subject 13 separately, an anatomical parcellation of left and right $S 1$ in native space (i.e. 14 anatomical S1 mask) was computed (Connectome Mapper 3). In addition, for each 15 hand separately, an F-contrast $(\mathrm{p}<0.0001$ uncorrected) across all stimulated hand regions was computed. The active voxels within the F-contrast were used as a functional S1 mask to identify all voxels responding to at least the stimulation of one hand region. Finally, t-contrasts (against rest) were also computed for each stimulated hand region. Then, based on a "winner takes all" approach, each voxel contained within the anatomical and functional S1 masks was labeled as representing the hand region whose stimulation elicited the highest t-score (against rest) for that particular voxel. This approach produces continuous and non-overlapping S1 maps comparable to phase encoding approaches used in mapping studies (Olman et al. 2010; SaadonGrosman et al. 2015; Sanchez-Panchuelo et al., 2012; Zeharia et al., 2015).

\subsection{Analysis of cortical distance}

Within each identified hand region representation, the coordinates of the peak activation (maximum t-value) were extracted. These 3D coordinates were transformed into indices of the nearest vertices on the surface space. The surface distances (geodesic) between PR and FRs were calculated for each participant using FreeSurfer

31 (mris_pmake). The statistical analysis described below (section 2.10) was conducted

32 to assess whether the cortical distance was increasing between PR and FRs as 33 expected by a serial somatotopic arrangement: "PALM-D1 > PALM-D2 > PALM-D3 > 34 PALM-D4 > PALM-D5 ".

\section{$35 \quad 2.7$ Analysis of co-activations}

36 To investigate the functional interactions between PR and FRs, we computed the co37 activations between PR and FRs. To this end, we computed the average BOLD 
1 response (beta values) within each FR during the stimulation of the palm ( $P->F R)$, as

2 well as the average BOLD response within PR during the stimulation of each finger (F-

3 >PR). Co-activations between $\mathrm{PR}$ and each FR were defined as the average between

$4 \quad \mathrm{P}->\mathrm{FR}$ and F->PR. This analysis was conducted in the native space of individual

5 subjects. The statistical analysis described below (section 2.10) was conducted to assess whether co-activations between PR and FRs reflected their somatotopic arrangement.

\subsection{Analysis of multi-voxel activity patterns}

10 To further investigate the functional interactions between PR and FRs, we compared 11 the multi-voxel activity patterns associated with palm and fingers stimulation 12 (Kriegeskorte et al, 2008). Compared to the analysis of co-activations, this measure 13 of functional interactions does not rely on the definition of hand region representations 14 associated with each body part stimulated. Separately for each participant and each 15 hand, we computed a GLM analysis to estimate the beta parameters associated with each period of tactile stimulation (24 tactile stimulation regressors and 6 rigid body motion regressors). Within the active voxels identified to define somatosensory hand representations (see section 2.5), the cross-validated (odd-even split across trials) Mahalanobis distance (Nili et al. 2014) between activity patterns associated with palm and fingers stimulation was computed as a measure of pattern dissimilarity. This analysis was conducted in the native space of individual subjects. The statistical analysis described below (section 2.10) was conducted to assess whether the multivoxel activity patterns associated with palm and fingers stimulation reflected the somatotopic arrangement of PR and FRs.

\subsection{Analysis of resting-state functional connectivity}

Compared to the analyses of co-activations and multi-voxel activity patterns, this measure quantifies functional interactions in the absence of tactile stimulation. Resting-state data were processed using the Conn toolbox (Withfield-Gabrieli et al., 2012). At each voxel, the BOLD signal was band-pass filtered $(0.008-0.09 \mathrm{~Hz})$. The cardiac and respiratory related components of the BOLD signal were estimated using the RETROICOR algorithm (Glover et al., 2000) and regressed out from the data. The average BOLD signal of white matter and cerebrospinal fluid (CSF) and the six estimated motion parameters were also included as nuisance regressors in the model.

35 The bivariate temporal correlations between PR and FRs were calculated from the 36 preprocessed BOLD time-courses of the resting state run. The obtained correlation 37 coefficients were transformed into gaussian values by applying the Fisher transform 
1 (Fisher, 1915). This analysis was conducted in the native space of individual subjects.

2 The statistical analysis described below (section 2.10) was conducted to assess

3 whether rs-FC between PR and FRs reflected their somatotopic arrangement.

\subsection{Statistical hypotheses and analyses}

First, we used Bayesian statistics to investigate the relationship between PR and FRs across fingers and across body side using the data obtained from the analyses of cortical distance, of co-activations, of multi-voxel activity patterns and of resting-state functional connectivity. Separately for each measure, we computed a two-way Bayesian repeated-measures ANOVA with "FINGER" (5 levels: P-D1, P-D2, P-D3, P-

11 D4 and P-D5) and "SIDE" (2 levels: right hand, $\mathrm{RH}$, and left hand, $\mathrm{LH}$ ) as within-subject 12 factors (JASP v0.13).

13 Second, we aimed at validating specific hypotheses regarding the somatotopic and 14 functional organization of hand representations. In particular, we directly tested the 15 hypothesis that PR and FRs are organized in serial arrangement in human S1 (see serial arrangement in Fig.1A, Moore et al., 2000; Rasmussen and Penfield, 1947). In addition, we speculated that such organization would not be reflected in the functional interactions between PR and FRs as PR would not interact preferentially with D5, then with D4, then with D3, then with D2 and least with D1 (Bullock and Dollar, 2011; Pont et al., 2009). To test these hypotheses, we used the R package bain (Hoijtink et al., 2019), which allows computing Bayesian statistics based on informative hypotheses (i.e. hypothesis driven tests). We computed Bayesian one-way repeated-measures ANOVAs separately for each measure (cortical distance, coactivations, multi-voxel activity patterns and resting-state functional connectivity) and each body side (right hand and left hand). We compared three hypotheses: 1) $\mathrm{H}_{1}$, a hypothesis of equivalence between the tested variables with a difference between pairs of variables smaller than a Cohen's $\mathrm{d}$ of 0.2 (P-D1 $\approx \mathrm{P}-\mathrm{D} 2 \approx \mathrm{P}-\mathrm{D} 3 \approx \mathrm{P}-\mathrm{D} 4 \approx \mathrm{P}-$ D5) (Sawilowsky, 2009), 2) $\mathrm{H}_{2}$, a hypothesis of ordering between the tested variables (P-D1 > P-D2 > P-D3 > P-D4 > P-D5 for cortical distance and pattern dissimilarity or $\mathrm{P}-\mathrm{D} 1<\mathrm{P}-\mathrm{D} 2$ < P-D3 < P-D4 < P-D5 for co-activations and functional connectivity), 3) and $\mathrm{H}_{\mathrm{u}}(\mathrm{P}-\mathrm{D} 1, \mathrm{P}-\mathrm{D} 2, \mathrm{P}-\mathrm{D} 3, \mathrm{P}-\mathrm{D} 4, \mathrm{P}-\mathrm{D} 5)$, the alternative unrestricted hypothesis (i.e. 32 the null hypothesis).

33 Finally, we computed a Bayesian regression between each measure of functional 34 interactions as observed variable (co-activations, multi-voxel activity patterns and 35 resting-state functional connectivity) and cortical distance as predictor variable (JASP 36 v0.13).

37 For each Bayesian test, we assumed equal prior probabilities and report the Bayes 
1 factors (BF) and posterior probabilities (PP). We considered Bayesian factors $>3$ as

2 positive evidence, $>10$ as strong evidence, $>30$ as very strong evidence (Kass and

3 Raftery, 1995).

4

\subsection{Dissimilarity analysis}

To further investigate the functional organization of hand representations, we conducted dissimilarity analysis (Akselrod et al., 2017; Kriegeskorte et al, 2008) and compared the representational geometry associated with the computed measures (cortical distances, co-activations, multi-voxel activity patterns and functional connectivity) with three models of hand representation. Separately for each subject and each hand, the four measures of dissimilarity (cortical distances, co-activations, multi-voxel activity patterns and functional connectivity) were computed between all pairs of fingers in addition to between each finger and the palm to form a $6 \times 6$ dissimilarity matrix. The computed dissimilarity matrices were compared with: 1) a model based on the physical shape of the hand, termed "Body" model; 2) a model of somatotopy reflecting the serial arrangements of FRs and PR, termed "Linear" model; 3) a control model, termed "Circular" model.

The "cortical distance" dissimilarity was computed as the surface distance between the coordinates of peak activations associated with the stimulated hand regions similarly to the analysis presented in section 2.6.

The "co-activation" dissimilarity was computed as the co-activations between pairs of $\mathrm{S} 1$ hand representations similarly to the analysis presented in section 2.7 . The coactivations between pairs of $\mathrm{S} 1$ hand representations correspond to a measure of similarity, i.e. pairs of $\mathrm{S} 1$ hand representations are considered similar if they are reciprocally co-activated when stimulated separately. The $6 \times 6$ similarity matrices of coactivations were transformed into $6 \times 6$ dissimilarity matrices by subtracting each coactivation value from the diagonal value of the same row (i.e. $b_{i, j}=c_{i, i}-c_{i, j}$, where $b$ represents the similarity matrix of co-activations, $c$ the dissimilarity matrix of coactivations, $i$ the row indices and $j$ the column indices).

The "multi-voxel activity pattern" dissimilarity was computed as the cross-validated mahalanobis distance between the multi-voxel patterns of $\mathrm{S} 1$ activations associated with the stimulated hand regions similarly to the analysis presented in section 2.8 .

The "functional connectivity" dissimilarity was computed based on the resting-state functional connectivity between pairs of $S 1$ hand representations similarly to the analysis presented in section 2.9. The resting-state functional connectivity is a measure of similarity, and it was transformed into a measure of dissimilarity by subtracting the bivariate correlation to 1 (i.e. 1-correlation). 
1 The "Body" model was computed using an independent dataset including 9 healthy

2 controls from a previous study (Mehring et al., 2019). The data consisted in a

3 localization task, where participants reported the perceived location of different parts

4 of their right hand including the tip and $2^{\text {nd }}$ knuckle of each finger, as well as the center

5 of the palm. The real position of these locations were also recorded. Using these data,

6 we computed average pair-wise distances between the fingers (defined as the average

7 location between the tip and the $2^{\text {nd }}$ knuckle) and the center of the palm. This resulted

8 in a single "Body" model (Fig.6A). The "Linear" model was conceived as a regular

9 decrease in similarity between each adjacent element of the matrix with a step of 1

10 (arbitrary unit), we note that this model corresponds to a serial model of S1 somatotopy

11 with perfect spacing between representations (Fig.6B). The "Circular model" was

12 conceived as a control model reflecting a plausible geometry of hand representations,

13 but not related to the body or to S1 somatotopy with the palm located in the center and

14 the fingers arranged radially around the palm (Fig.6C).

15 The matrices corresponding to the four measures of dissimilarity were correlated with 16 the matrices corresponding to the three models separately for each participant (upper 17 part of the matrices were treated as data vectors). For each measure, an upper bound 18 limit of maximum correlation (i.e. noise ceiling) was calculated as the correlation 19 between each subject's dissimilarity matrix and the group average dissimilarity matrix, 20 averaged across subjects. In order to compare the different models, the resulting 21 correlation values were normalized using the Fischer transformation and statistically 22 analyzed using Bayesian paired t-tests between the model with the highest correlation 23 and the other two models (JASP v0.13). We considered Bayesian factors $>3$ as 24 positive evidence, $>10$ as strong evidence, $>30$ as very strong evidence (Kass and 25 Raftery, 1995).

26 For display purposes, we used classical multidimensional scaling (also known as 27 Principal Coordinate Analysis, Cooper and Seber, 1985) to represent the models and the dissimilarity measures on a 2D plot.

\subsection{Data and code availability statement}

31 The final data presented in the Results section (cortical distances, co-activations, 32 multi-voxel patterns, resting-state functional connectivity and dissimilarity analysis) are openly available on the Zenodo platform. Data analyses were carried out using publicly available resources and/or are fully reproducible from the information provided in the

35 Methods section. Raw data will not be shared to guarantee privacy and confidentiality 36 for the participants. 
bioRxiv preprint doi: https://doi.org/10.1101/2020.09.07.286062; this version posted September 9, 2020. The copyright holder for this preprint (which was not certified by peer review) is the author/funder, who has granted bioRxiv a license to display the preprint in perpetuity. It is made available under aCC-BY-NC 4.0 International license.

\section{3. RESULTS}

2 Twelve hand regions ( 6 on each hand) were stimulated during fMRI acquisition to map 3 their cortical representations within S1. This led to a total of 36 mapped 4 representations in S1 per subject (see Methods). Visual inspection of individual maps 5 suggested that the palm (i.e. PRs) was located medially with respect to the D5 FR in 6

7 all participants. The $\mathrm{S} 1$ hand maps of a representative subject are shown in Fig.1 and

8 S1 hand maps for all subjects are shown in supplementary materials (Fig.S1-S2).

\section{$10 \quad 3.1$ Cortical distance}

11 We compared the geodesic distance between PR and each FR using Bayesian 12 statistics (see section 2.10). As shown in Fig.2, the distance between PR and each FR

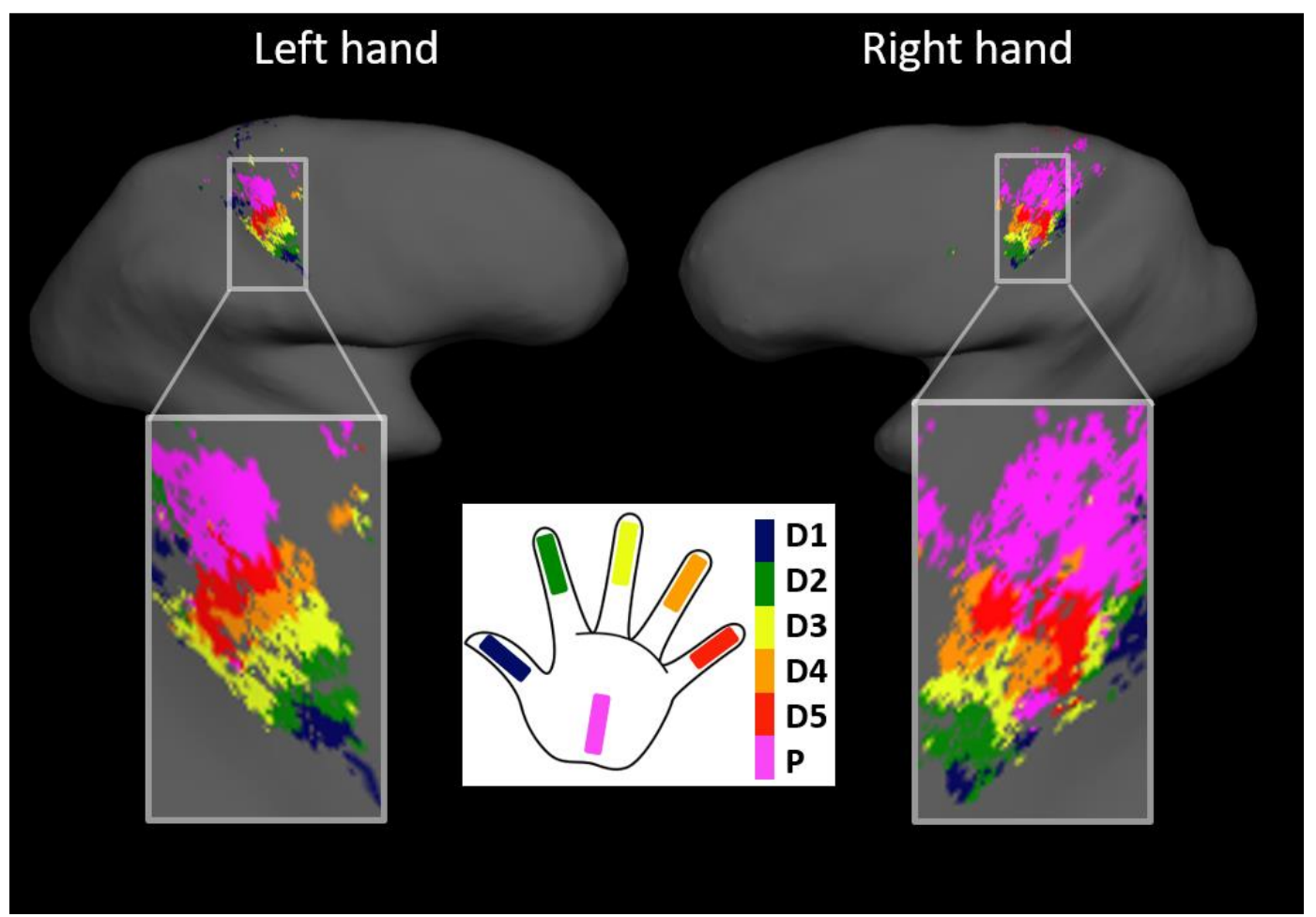

Figure 1: Palm to fingers somatotopy. $\mathrm{S} 1$ hand map of a representative subject suggesting the following arrangement in humans: D1 - D2 - D3 - D4 - D5 - PALM.

13 is decreasing when moving from P-D1 to P-D5.

14

15 The two-way ANOVA showed a main effect of finger $\left(B F=2.011 \mathrm{e}^{+18}, \mathrm{PP}=0.866\right)$, a main

16 effect of body side $(B F=3396.92, P P=0.866)$, but no interaction $(B F=0.154, P P=0.134)$. 

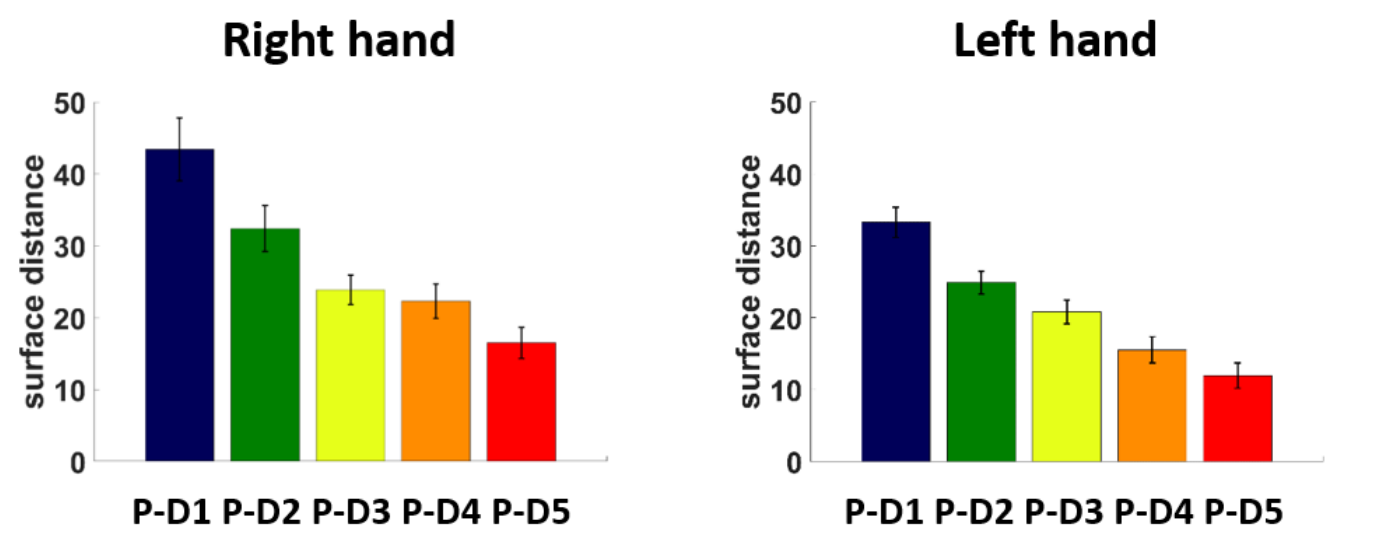

Figure 2. Cortical distance. Bar plots of the cortical distances between PR and each FR in the left hemisphere (right hand representations) and in the right hemisphere (left hand representations). Error bars represent the standard error of the mean.

1 The main effect of body side, with very strong evidence $(B F>100)$, was due to reduced

2 distances for left hand representations compared to the right hand.

3 The hypothesis driven ANOVAs strongly supported the ordering hypothesis, $\mathrm{H}_{2}$, for 4 both hands, suggesting a latero-medial serial arrangement, with the palm located after 5 D5: "D1 - D2 - D3 - D4 - D5 - PALM" (right hand: BF=25.487, PP=0.963; left hand: $\mathrm{BF}=38.73, \mathrm{PP}=0.975$, see $\underline{\mathrm{Tab} .1}$ ).

\begin{tabular}{|l|c|c|c|c|}
\hline & \multicolumn{2}{|c|}{ Right Hand } & \multicolumn{2}{c|}{ Left Hand } \\
\hline & $\mathrm{BF}$ & $\mathrm{P}(\mathrm{H})$ & $\mathrm{BF}$ & $\mathrm{P}(\mathrm{H})$ \\
\hline $\mathrm{H}_{1}$ (equivalence): $\mu_{1} \approx \mu_{2} \approx \mu_{3} \approx \mu_{4} \approx \mu_{5}$ & 0.0 & 0.0 & 0.0 & 0.0 \\
\hline $\mathrm{H}_{2}$ (ordering): $\mu_{1}>\mu_{2}>\mu_{3}>\mu_{4}>\mu_{5}$ & 25.87 & $\mathbf{0 . 9 6 3}$ & 38.73 & $\mathbf{0 . 9 7 5}$ \\
\hline $\mathrm{H}_{\mathrm{u}}$ (unrestricted): $\mu_{1}, \mu_{2}, \mu_{3}, \mu_{4}, \mu_{5}$ & & 0.037 & & 0.025 \\
\hline
\end{tabular}

Table 1. Bayesian statistics on cortical distance. Bayesian ANOVAs were conducted for each hand separately. The Bayes Factor and posterior probability are reported for each tested hypothesis.

7 To summarize, the analysis of cortical distances comprehensively suggests a serial

8 latero-medial arrangement, with the palm being represented most laterally in human

9 S1. In addition, we found reduced cortical distances between PR and FRs for left hand representations.

11 
1 We then analyzed the co-activations between PR and FRs, which assess how strongly

2 these representations are reciprocally co-activated when stimulated separately. As

3 shown in Fig.3, there was no consistent evidence of an ordering effect, rather the

4 strongest co-activations are found between P-D1 and P-D5.

5 The two-way ANOVA showed a main effect of finger ( $B F=12140.48, P P=0.946)$, a

Right hand

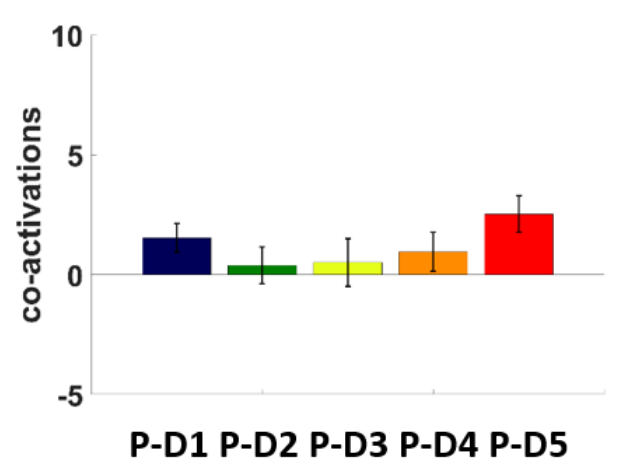

Left hand

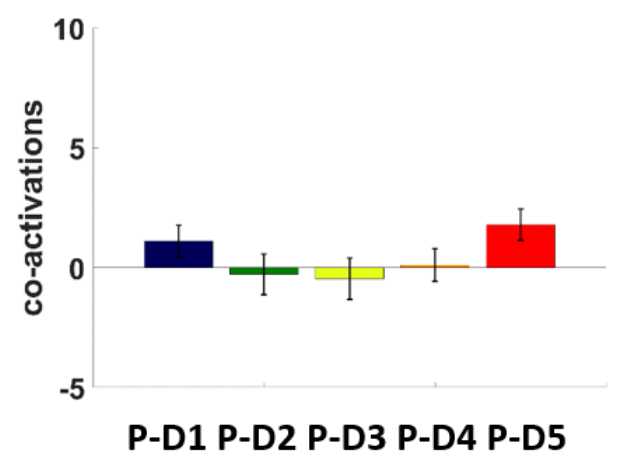

Figure 3. Co-activations. Bar plots of the co-activations between PR and each FR for the right hand (represented in the left hemisphere) and for the eft hand (represented in the right hemisphere). Error bars represent the standard error of the mean.

6 main effect of body side ( $\mathrm{BF}=4.84, \mathrm{PP}=0.784$ ), but no interaction ( $\mathrm{BF}=0.069$, $P P=0.054)$. The main effect of body side, with positive evidence $(B F>3)$, was due to reduced co-activations for left hand representations compared to the right hand. We note that this is effect is not consistent with the effect of reduced cortical distances for left hand representations, which would rather predict stronger functional interactions with reduced distances.

\begin{tabular}{|l|c|c|c|c|}
\hline & \multicolumn{2}{|c|}{ Right Hand } & \multicolumn{2}{c|}{ Left Hand } \\
\hline & $\mathrm{BF}$ & $\mathrm{P}(\mathrm{H})$ & $\mathrm{BF}$ & $\mathrm{P}(\mathrm{H})$ \\
\hline $\mathrm{H}_{\mathbf{1}}$ (equivalence): $\boldsymbol{\mu}_{\mathbf{1}} \approx \boldsymbol{\mu}_{\mathbf{2}} \approx \boldsymbol{\mu}_{\mathbf{3}} \approx \boldsymbol{\mu}_{\mathbf{4}} \approx \boldsymbol{\mu}_{\mathbf{5}}$ & 0.0 & 0.0 & 0.0 & 0.0 \\
\hline $\mathrm{H}_{\mathbf{2}}$ (ordering): $\boldsymbol{\mu}_{\mathbf{1}}>\boldsymbol{\mu}_{\mathbf{2}}>\boldsymbol{\mu}_{\mathbf{3}}>\boldsymbol{\mu}_{\mathbf{4}}>\boldsymbol{\mu}_{\mathbf{5}}$ & 0.290 & 0.225 & 0.042 & 0.040 \\
\hline $\mathrm{H}_{\mathrm{u}}$ (unrestricted): $\boldsymbol{\mu}_{\mathbf{1}}, \boldsymbol{\mu}_{\mathbf{2}}, \boldsymbol{\mu}_{\mathbf{3}}, \boldsymbol{\mu}_{\mathbf{4}}, \boldsymbol{\mu}_{\mathbf{5}}$ & & $\mathbf{0 . 7 7 5}$ & & $\mathbf{0 . 9 6 0}$ \\
\hline
\end{tabular}

Table 2. Bayesian statistics on co-activations. Bayesian ANOVAs were conducted for each hand separately. The Bayes Factor and posterior probability are reported for each tested hypothesis.

12 The hypothesis driven ANOVAs supported the unrestricted hypothesis, $H_{0}$, for both

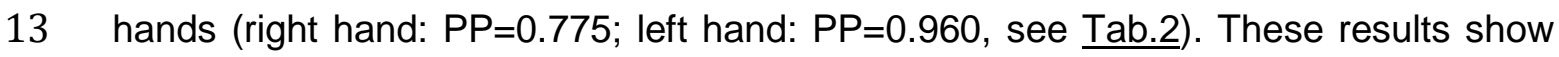
14 that co-activations between PR and FRs do not follow a pattern predicted by the 15 somatotopic organization and do not show equivalent interactions between PR and the 
1 five FRs. Finally, we did not find evidence for a relationship between co-activations

2 and cortical distances $(\mathrm{BF}=0.484, \mathrm{PP}=0.326)$.

4 To summarize, these analyses suggest that functional interactions between PR and

5 FRs, as measured by the degree of mutual co-activations during isolated stimulation,

6 are not equivalent between the PR and each other finger, but rather that that PR might

$7 \quad$ interact preferentially with some FRs, namely D1 and D5. They also do not reflect the

8 somatotopic sequence in S1, suggesting that if a specific pattern of interaction

9 between PR and FRs exist, it does not follow the somatotopic organization. These

10 results might suggest the presence of other patterns of functional interactions that were

11 not formulated in our hypotheses, and therefore, to address this point, we performed

12 dissimilarity analysis that is presented below (3.5).

\subsection{Multi-voxel activity patterns}

15 We then compared the dissimilarity (i.e. mahalanobis distance) between multi-voxel activity patterns in $\mathrm{S} 1$ associated with the tactile stimulation of the palm and of the five

17 fingers. As shown in Fig.5, the highest dissimilarity was observed between P-D3 for 18 both hands, while the lowest dissimilarity was observed between palm-D1 and palm19 D5 for both hands. This result is compatible with the co-activation pattern between PR and FRs (i.e. more interaction/similarity between P-D1 and P-D5).
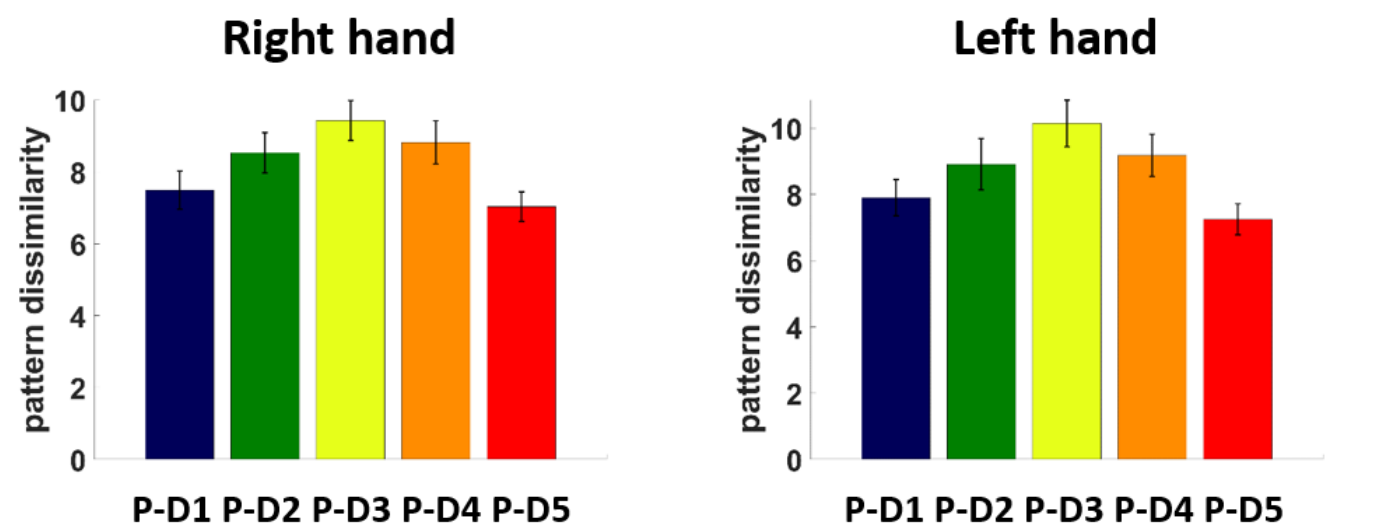

Figure 4. Multi-voxel activity patterns. Bar plots of the dissimilarity between multivoxel activity patterns associated with the stimulation of the palm and of each finger in the left hemisphere (right hand representations) and in the right hemisphere (left hand representations). Error bars represent the standard error of the mean.

22 The two-way ANOVA showed a main effect of finger ( $B F=3.796 \mathrm{e}^{+7}, \mathrm{PP}=0.973$ ), but no 23 main effect of body side $(B F=0.681, P P=0.394)$ and no interaction $(B F=0.069$, $24 \mathrm{PP}=0.027)$.

25 The hypothesis driven ANOVAs supported the unrestricted hypothesis, $H_{0}$, for both 
1 hands (right hand: $\mathrm{PP}=1.0$; left hand: $\mathrm{PP}=1.0$, see $\underline{\mathrm{Tab} .3}$ ). These results show that 2 multi-voxel activity patterns do not follow a pattern predicted by the somatotopic 3 organization and do not follow a pattern of equivalence between the palm and the 4 fingers. Finally, we did not find evidence for a relationship between co-activations and 5 cortical distances $(\mathrm{BF}=0.187, \mathrm{PP}=0.158)$.

\begin{tabular}{|c|c|c|c|c|}
\hline & \multicolumn{2}{|c|}{ Right Hand } & \multicolumn{2}{|c|}{ Left Hand } \\
\hline & BF & $\mathrm{P}(\mathrm{H})$ & $\mathrm{BF}$ & $\mathbf{P}(\mathrm{H})$ \\
\hline$H_{1}$ (equivalence): $\mu_{1} \approx \mu_{2} \approx \mu_{3} \approx \mu_{4} \approx \mu_{5}$ & 0.0 & 0.0 & 0.0 & 0.0 \\
\hline$H_{2}$ (ordering): $\mu_{1}>\mu_{2}>\mu_{3}>\mu_{4}>\mu_{5}$ & 0.0 & 0.0 & 0.0 & 0.0 \\
\hline$H_{u}$ (unrestricted): $\mu_{1}, \mu_{2}, \mu_{3}, \mu_{4}, \mu_{5}$ & & 1.0 & & 1.0 \\
\hline
\end{tabular}

Table 3. Bayesian statistics on multi-voxel activity patterns. Bayesian ANOVAs were conducted for each hand separately. The Bayes Factor and posterior probability are reported for each tested hypothesis.

8 To summarize, these analyses suggest that functional interactions between PR and

9 FRs, as measured by multi-voxel activity pattern dissimilarity, do not reflect equivalent 10 interactions between the palm and the five fingers and do not reflect the somatotopic 11 sequence in S1. Consistent with the results of the co-activations analysis (section 3.2), 12 we observed for both hands that minimal pattern dissimilarity was found between P. 13 D1 and P-D5, possibly suggesting the presence of yet another pattern of functional 14 interactions between PR and FRs that was not formulated in our hypotheses (see 15 dissimilarity analysis, 3.5).

\section{$17 \quad 3.4$ Resting-state functional connectivity}

18 Finally, we compared the functional connectivity between PR and FRs. As shown in 19 Fig.6, there is a qualitative trend towards stronger functional connections between PR 20 and FRs which are located closer to PR in S1, although this pattern is not fully

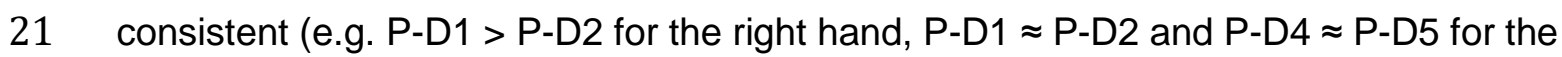
22 left hand). 
Right hand

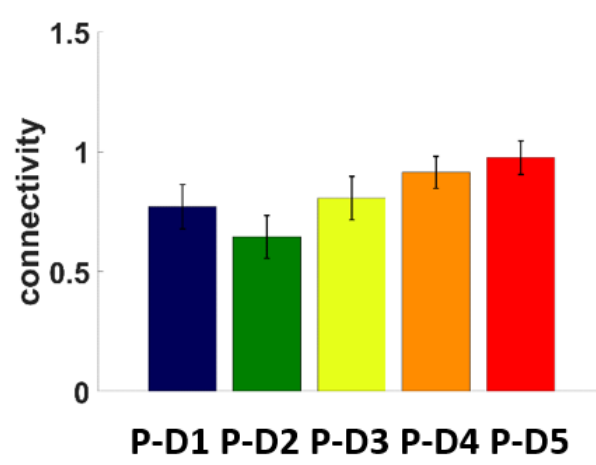

Left hand

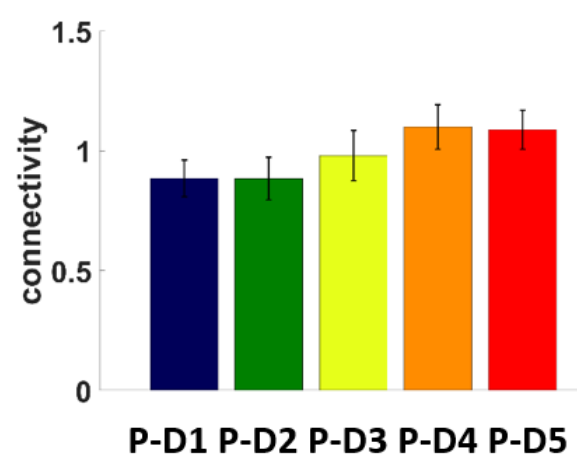

Figure 5. Functional connectivity. Bar plots of the functional connectivity (Z-score) between PR and each of the FR in the left hemisphere (right hand representations) and in the right hemisphere (left hand representations). Error bars represent the standard error of the mean.

2 The two-way ANOVA showed a main effect of finger ( $B F=113.943, P P=0.914)$, a main

3 effect of body side ( $B F=153.667, P P=0.916)$, but no interaction ( $B F=0.086, P P=0.078)$.

4 The main effect of body side, with very strong evidence (BF>100), was due to reduced

5 functional connectivity for right hand representations compared to the left hand. We

6 note that this is effect is consistent with the effect of reduced cortical distances for left

7 hand representations, which would predict stronger functional interactions with

8 reduced distances.

9 The hypothesis driven ANOVAs supported the ordering hypothesis, $H_{2}$, for both hands 10 (right hand: $B F=1.235, P P=0.553$; left hand: $B F=44.70, P P=0.978$, see $\underline{T}$ ab.4). We 11 note that, for the right hand, low positive evidence was found for the ordering 12 hypothesis, $H^{2}$. These results show that the functional connectivity between PR and 13 FRs follows, at least to some extent, a pattern predicted by the somatotopic 14 organization.

\begin{tabular}{|l|c|c|c|c|}
\hline & \multicolumn{2}{|c|}{ Right Hand } & \multicolumn{2}{c|}{ Left Hand } \\
\hline & $\mathrm{BF}$ & $\mathrm{P}(\mathrm{H})$ & $\mathrm{BF}$ & $\mathrm{P}(\mathrm{H})$ \\
\hline $\mathrm{H}_{\mathbf{1}}$ (equivalence): $\boldsymbol{\mu}_{1} \approx \boldsymbol{\mu}_{2} \approx \mu_{3} \approx \mu_{4} \approx \mu_{5}$ & 0.0 & 0.0 & 0.0 & 0.0 \\
\hline $\mathrm{H}_{2}$ (ordering): $\mu_{1}>\mu_{2}>\mu_{3}>\mu_{4}>\mu_{5}$ & 1.235 & $\mathbf{0 . 5 5 3}$ & $\mathbf{4 4 . 7 0}$ & $\mathbf{0 . 9 7 8}$ \\
\hline $\mathrm{H}_{\mathrm{u}}$ (unrestricted): $\mu_{1}, \mu_{2}, \mu_{3}, \mu_{4}, \mu_{5}$ & & 0.447 & & 0.022 \\
\hline
\end{tabular}

Table 4. Bayesian statistics on functional connectivity. Bayesian ANOVAs were conducted for each hand separately. The Bayes Factor and posterior probability are reported for each tested hypothesis. 
1 Finally, we found very strong evidence for a relationship between functional

2 connectivity and cortical distances with stronger functional connectivity being

3 associated with reduced cortical distance (BF=1058.725, $\mathrm{PP}=0.999)$.

5 To summarize, these functional connectivity results suggest that functional interactions 6 between PR and FRs, as measured by resting-state functional connectivity, reflect, at 7 least partially, the somatotopic sequence in $\mathrm{S} 1$. This is further supported by the strong 8 evidence for a negative relationship between functional connectivity and cortical 9 distance.

\subsection{Dissimilarity analysis}

12 Considering that, with the exception of functional connectivity, the measure of 13 functional interactions between PR and FRs were not associated with the somatotopic 14 ordering hypothesis nor with the equivalence hypothesis, we extended previous analyses to investigate the representational geometry of PR and FRs associated with each of the computed measures (cortical distances, co-activations, multi-voxel activity patterns and functional connectivity). We computed dissimilarity matrices based on these four measures and compared them with three models of hand representation, the "Body" model, the "Linear" model and the "Circular" model. Fig.6 shows the four models $(A-C)$ and the three dissimilarity measures (D-G) with their corresponding 2D configuration computed with multidimensional scaling. We note that in this analysis, similar results were obtained for both hands, thus the data were averaged across both hands. Separate data for right and left hands are shown in supplementary materials (Fig.S3-S4).

25 The three models were designed to capture different aspects of what S1 could represent. The "Body" model formed a 2D configuration compatible with the shape of a hand. The "Linear" model formed a 2D configuration compatible with the somatotopic sequence "D1-D2-D3-D4-D5-PALM". Finally, the "Circular" model formed a 2D configuration corresponding to a plausible geometry of hand representations, but different from the real shape of a hand and different from $\mathrm{S} 1$ hand somatotopic

31 organization.

32 To assess which model best described the four dissimilarity measures (cortical 33 distances, co-activations, multi-voxel activity patterns and functional connectivity), we 34 computed the correlation between each dissimilarity matrix and the three models and 35 computed Bayesian paired t-tests across these correlations to identify the best models 36 (Tab.S1). For cortical distance, we found that the "Linear" model was the best $37(r=0.80 \pm 0.13)$ and outperformed the other models with very strong evidence (Linear $\neq$ 
1 Body: $B F=173.96$, Linear $\neq$ Circular: $B F=22670.11)$. For co-activations, we found that

2 the "Body" model was the best $(r=0.77 \pm 0.06)$ and outperformed with very strong 3 evidence the "Linear" and "Circular" models (Body $\neq$ Linear: BF $=2.350 \mathrm{e}^{+6}$, Body $\neq$

4 Circular: $B F=7714.05)$. For multi-voxel activity patterns, we found that the "Body" 5 model was the best $(r=0.71 \pm 0.11)$, outperformed the "Linear" model with very strong 6 evidence (Body $\neq$ Linear: $B F=16619.11)$ and outperformed with positive evidence the 7 "Circular" model (Body $\neq$ Circular: BF = 3.77). Finally, for functional connectivity, we 8 found that the "Linear" model was the best $(r=0.62 \pm 0.20)$, outperformed the "Body" 9 model with positive evidence (Linear $\neq$ Body: BF=4.67) and outperformed to "Circular" 10 model with very strong evidence (Linear $\neq$ Circular: $B F=73.00)$.

11 As a control analysis, to confirm that the aforementioned results cannot be explained 12 by the variance associated with the fingers only, we replicated the whole dissimilarity 13 analysis by excluding the palm from the data, leading to $5 \times 5$ dissimilarity matrices 14 across the 5 fingers. First, we found that the variance explained by the best models 15 was similar when considering the palm and the five fingers or when considering only the five fingers (Fig.S5). However, when only considering the five fingers the analysis could not disambiguate between the "Body" and "Linear" models. Thus, only when considering the palm and the fingers together, it is possible to highlight a double dissociation between dissimilarity measures best matching the models related to the shape of a hand (co-activations and multi-voxel activity patterns) and dissimilarity measures best matching the model related to somatotopy (cortical distances and functional connectivity).

To summarize, dissimilarity analysis showed that co-activations and multi-voxel activity patterns were related to the shape of a hand, while cortical distances and functional connectivity were rather related to somatotopy. This shows that the representational geometry of hand functional interactions (with the exception of functional connectivity) matched the physical structure of the hand rather than the somatotopic organization of hand representations. 


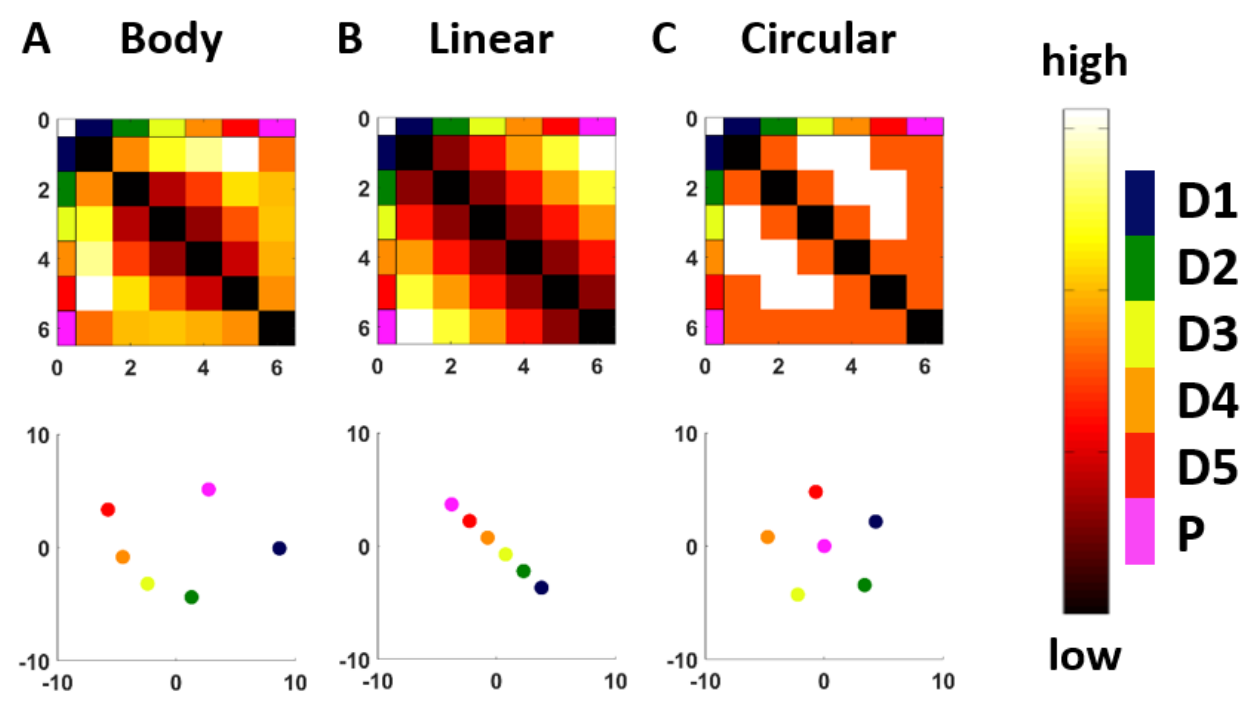

D Cort. Dist.
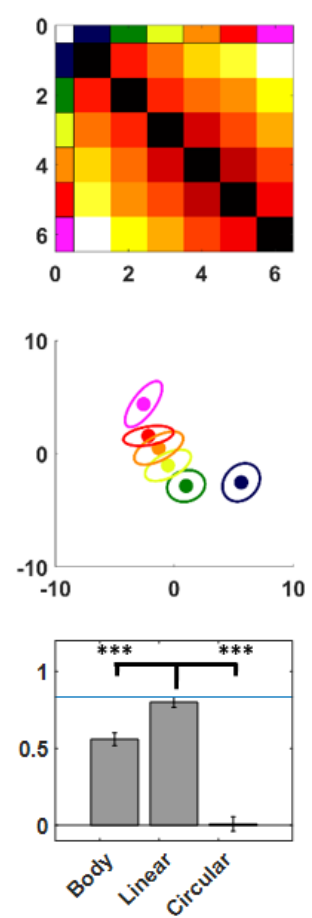

\section{E Co-activ.}
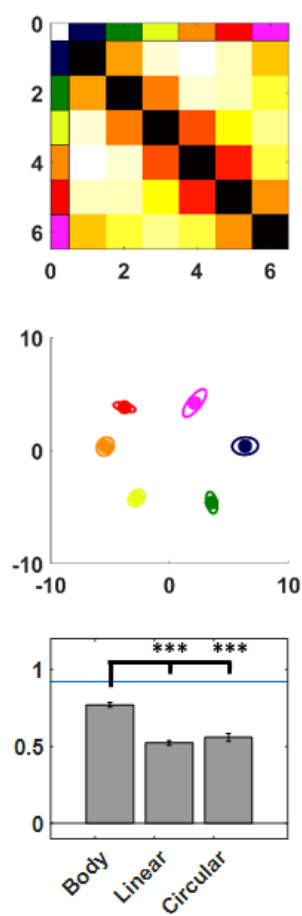

F Multiv. Pat.
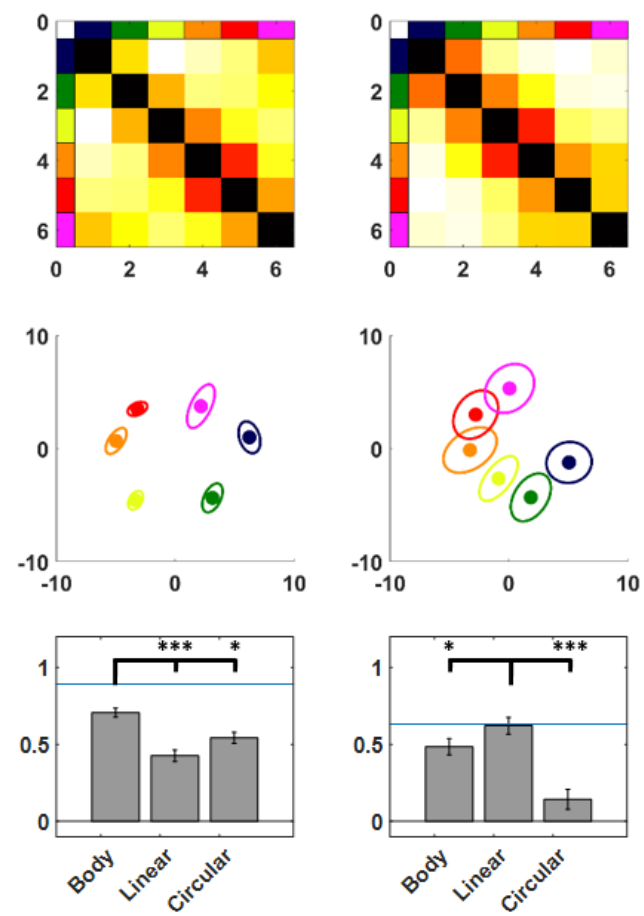

Figure 6. Dissimilarity analysis. A-C. Dissimilarity matrix and 2D configuration for the "Body", "Linear" and "Circular" models. D-G. Dissimilarity matrix and 2D configuration for the dissimilarity measures based on cortical distances, coactivations, multi-voxel activity patterns and functional connectivity. The correlations between each dissimilarity measure and the three models are shown in the corresponding bar plots. Asterisks indicate the level of evidence found across Bayesian comparisons. The blue line indicates the noise ceiling. Data presented here are averaged across hands. 


\section{4. DISCUSSION}

2 The present study aimed at investigating PR in human $\mathrm{S} 1$ and its interactions with the five FRs, by analyzing (1) the cortical distances between somatotopic representations (cortical distance), (2) how PR and FRs co-activate during tactile stimulation (coactivation), (3) the similarity between activity patterns during tactile stimulation (multivoxel activity pattern), and (4) how PR and FRs are functionally connected to each other (functional connectivity). During the acquisition of $\mathrm{fMRI}$ data at ultra-high field (7T), six hand regions (D1 - D2 - D3 - D4 - D5 - PALM) on each side of the body were stimulated using natural touch in a group of healthy subjects. This allowed us to identify the tactile representations of the stimulated hand regions within S1. First, we demonstrated the serial arrangement of the somatotopic sequence: D1 - D2 - D3 - D4 - D5 - PALM. Second, we found that this somatotopic sequence is not reflected in the pattern of functional interactions between PR and FRs, with the exception of functional connectivity (see below). Instead, the representational geometry of functional interactions between hand representations better matches the physical shape of the hand rather than the somatotopic organization of its representations.

\subsection{Mismatch between S1 hand somatotopy and hand structure in humans}

The results obtained from the analysis of cortical distances between PR and FRs confirm that the palm representations in human $\mathrm{S} 1$ are located medially with respect to the representations of D5, corresponding to a serial somatotopic arrangement in S1 (Blankenburg et al., 2003; Moore et al., 2000; Rasmussen and Penfield, 1947). This layout does not correspond to the radial distribution of fingers along the palm on the body, thus creating a discontinuity between S1 hand somatotopy and the physical structure of the hand in humans. Discontinuities between somatotopy and body structure have been well documented in primates (Felleman et al., 1983; Kaas et al., 1979; Merzenich et al. 1978; Nelson et al. 1980; Rasmussen and Penfield, 1947; Sur et al., 1982). In particular, the latero-medial arrangement of fingers (D1 - D2 - D3 - D4 - D5) in S1, which is found in all primates, forms a hand-arm discontinuity with the latero-medial arrangement of the rest of the arm (distal to proximal). Interestingly, different somatotopic layouts of the pads (i.e. distal palm and base of the fingers) and fingers have been observed in S1 across primate species (Felleman et al., 1983; Merzenich et al., 1978; Nelson et al., 1980; Sur et al., 1982). In Owl and Squirrel Monkeys, the representations of the pads are included in FRs as their most proximal part (Merzenich et al., 1978; Sur et al., 1982). This is also the case in humans (Blankenburg et al., 2003; Sanchez-Panchuelo et al. 2012, 2014;

37 Schweisfurth et al., 2011, 2014). Contrastingly, in Cebus and Macaque Monkeys, the 
1 representations of the pads lie medially to D5 (Felleman et al., 1983; Nelson et al.,

2 1980). Thus, the location of the separation forming the somatotopic hand-arm

3 discontinuity appears to vary across primate species. Furthermore, this somatotopic

4 polymorphism does not correspond to phylogenetic relations between primate species

5 (Springer et al., 2012), possibly indicating that a conversion of hand somatotopic

6 layout may have occurred several times during primate evolution. Based on results

7 from this study and previous studies in primates, the proximal part of the palm is

8 represented medially with respect to the 5 fingers in S1 in all studied primate species

9 (present study and Blankenburg et al., 2003; Felleman et al., 1983; Merzenich et

10 al., 1978; Moore et al., 2000; Nelson et al., 1980; Rasmussen and Penfield, 1947;

11 Sur et al., 1982). Thus, the proximal part of the palm can be considered a reliable

12 landmark in S1 to study somatotopy within and across primate species.

\subsection{Mismatch between $\mathrm{S} 1$ hand somatotopy and $\mathrm{S} 1$ hand functional interactions} in humans

Previous studies focusing on fingers reported that $\mathrm{S} 1$ functional interactions between FRs, as measured by co-activations, followed a pattern compatible with $\mathrm{S} 1$ somatotopy, i.e. the adjacency between FRs in S1 predicts the degree of coactivations (Besle et al., 2014; Martuzzi et al., 2014). Similarly, a study focusing on motor representations of fingers showed that multi-voxel activity patterns in S1 associated with finger movements are well described by a somatotopic model of finger adjacency (Ejaz et. al, 2015), although these patterns were best described by the natural statistics of hand usage. This suggests that when considering FRs only, a consistency is found between the finger sequence on the hand, the finger somatotopy in $\mathrm{S} 1$, and finger functional interactions in $\mathrm{S} 1$. Our analyses of functional interactions between PR and FRs tested whether the S1 palm-to-fingers somatotopy predicts palm-to-fingers functional interactions, as measured by co-activations, multi-voxel activity patterns and resting-state functional connectivity. Importantly, considering the natural usage of the palm in synergy with the fingers for hand function, there is no a priori reason to expect stronger interactions between PR and FRs located closer to PR in S1 (e.g., between palm and D5). Concerning resting-state functional connectivity, dissimilarity analysis showed that functional connectivity matched better with the model reflecting S1 somatotopy. This result can be explained by the well-documented influence of cortical distance on resting-state functional connectivity in both topographically and non-topographically organized brain areas (Alexander-Bloch et al., 2013; Ercsey-Ravasz et al., 2013;

37 Raemakers et al., 2014; Salvador et al., 2005). This suggests a possible confound 
1 resulting from the use of resting-state functional connectivity to investigate the

2 relationship between functional interactions and cortical topography. More

3 interestingly, our statistical analyses of co-activations and multi-voxel activity patterns revealed that functional interactions between PR and FRs, indeed, do not reflect S1 palm-to-fingers somatotopy. Our analyses of co-activations highlighted that the palm interacts most strongly with D1 and D5. Similarly, multi-voxel activity patterns suggested that palm stimulation induced activity patterns most similar to D1 and D5 stimulation. This pattern of functional interactions is compatible with the physical shape of the hand where, at rest, the tips of D1 and D5 are closer to the center of the palm compared to other fingers. This view was further supported by dissimilarity analysis showing that the representational geometry of hand representations in S1 matched better with the models reflecting the shape of a hand (except for functional connectivity, see below). Another possibility is that the natural statistics of tactile experience during daily life leads to increased likelihood of palm-D1 and palm-D5 co-stimulation (Ejaz et. al, 2015). Whether the observed associations (palm-D1 and palm-D5) are better explained by the statistics of use-related tactile stimulation on the hand rather than simply the physical shape of the hand remains to be investigated. Note, however, that natural statistics of tactile stimulation depends on the hand structure, which would make the two hypotheses complementary.

\subsection{Differences between the right and left hands}

22 Our data also revealed interesting differences between the dominant right hand and the non-dominant left hand in our right-handed participants. We found that the righthand has overall larger distances between PR and FRs, which might suggest larger cortical territories in $\mathrm{S} 1$ for the dominant right hand. However, the inter-digit distances did not differ between right and left hands (Fig.S6), thus suggesting that the aforementioned effect is rather due to PR being located further away from FRs. This is in line with previous $\mathrm{fMRI}$ reports showing no difference in size between right and left finger representations (Boakye et al., 2000; Schweisfurth et al., 2018). Second, we found overall stronger co-activations between PR and FRs for the right hand compared to the left hand. This effect was not due to simple increased activations, as we found similar strength of activations (within representations) when comparing right and left hand representations (Fig.S7), which corroborates findings from previous fMRI studies showing no difference in strength of activations between right and left hands

35 (Boakye et al., 2000; Schweisfurth et al., 2018; but see Jung et al., 2003, 2008). 36 Rather, this points towards increased integrative properties between PR and FRs 37 during tactile stimulation. Finally, we also found reduced functional connectivity 
1 between PR and FRs for the right hand compared to the left hand, which might be

2 interpreted as increased independence in the absence of stimulation. While this seems

3 counterintuitive with respect to the previous result (increased co-activations during

4 stimulation), this might also suggest a context dependent tuning of integrative

5 properties for the dominant right hand (Di et al., 2013; Morgan and Price, 2004).

6 Results from the present and previous studies (Boakye et al., 2000; Schweisfurth et al., 2018) suggest overall no difference between right and left hand representations regarding basic functional properties (extent or strength of activations). This is compatible with behavioural observations showing no difference between right and left

10 hands in tactile spatial acuity (Sathian and Zangaladze, 1996). However, our data

11 suggest the presence of differences between the representaiton of the right and the

12 left hand related to integrative properties of somatosensory processing, which might 13 be linked to differences in dexterity associated with hand dominance (Andersen and 14 Siebner, 2018).

\subsection{Plasticity in topographically organized sensory areas}

17 It is believed that topographically organized cortical sensory maps evolved as an 18 optimal solution for energy-efficient spatio-temporal computations (Kaas, 1997). It is 19 currently accepted that topographic maps are shaped by a combination of at least two 20 different factors. First, during development, the axonal pathways from the skin to the 21 cortex are established through molecular matching interactions, which is governed by genetics (Udin and Fawcett, 1988). The formation of a prototypic topography of sensory maps during development would explain why individuals from a same species share a common architecture. Second, during daily life experience, the spatio-temporal receptive fields of neuronal populations are tuned by sensory stimulation and associated synaptic plasticity (Buonomano and Merzenich, 1998).

Our results provide an important account of mismatch between functional interactions and topographical organization. This supports the view that functional cortical interactions, that are consistent with the peripheral structure of the sensory space, can emerge despite the mismatch between topographical organization and the structure of the sensory space. However, our data could not disambiguate between possible contributions of the structure of the sensory space (i.e. the shape of the hand) and of the natural statistics of tactile stimulation received during everyday life (Ejaz et al., 34 2015) in shaping the functional interactions between hand representations. 35 Nevertheless, these two factors are by definition impossible to disentangle in normal 36 conditions, because the physical structure of the body directly impacts the pattern of natural stimulation during everyday life interactions. 
1 Crucially, topographic maps require the continuous competition and interaction

2 between inputs to maintain a normal organization, which is naturally provided during 3 activities of daily living (Buonomano and Merzenich, 1998). An extreme example of 4 plasticity in adult primary somatosensory areas is observed following limb amputation 5 (Flor et al., 1995; Kaas et al., 1983; Makin et al., 2013; Makin and Flor, 2020; 6 Serino et al., 2017) or to a lesser extent following limb immobilization (Langer et al., 2012; Liepert et al., 1995; Zannette et al., 1997). Furthermore, it has been shown that even a brief exposition to repeated sensory stimulation can induce plasticity in primary somatosensory areas (Godde et al., 1996; Muret et al., 2016; Pleger et al., 2001, 2003). Similar observations are found for the visual system and the auditory

11 system (Bilecen et al., 2000; Chino et al., 1992; Kaas et al., 1990; Kaas, 1991; 12 Merabet and Pascual-Lenoe, 2010; Syka et al., 2002). Considering the results of the 13 present study in light of the capacity of sensory areas to adapt to changes in the 14 structure of the sensory space, a consistency between somatotopic organization and functional interactions could be expected. A possible explanation is that a certain degree of flexibility in the consistency between topographic organization and functional interactions in sensory areas is tolerated. In other words, the metabolic energy cost to tolerate such mismatch is lower than the energy cost required for reorganization. This might suggest that processing mismatched sensory inputs would lead to reorganization only in case of substantial inconsistency.

\subsection{Study Limitations}

23 We provided tactile stimulation by means of manual stroking delivered by a human experimenter, thus introducing inherent variability in the timing, intensity and extent of stimulation. This choice was motivated by previous work from our group, showing that natural touch is able to induce reliable activations in S1 (Martuzzi et al., 2014; Akselrod et al., 2017; Serino et al., 2017), and stronger activations compared to mechanical stimulation (van der Zwaag et al., 2015). Although the increased variability associated with natural touch might contribute to the increased signal quality, the lack of controllability might have introduced systematic biases towards a specific body part. Thus, we cannot exclude that at least part of the variance explained by our results might be attributed to the lack of controllability of natural touch.

\section{CONCLUSIONS}

The present study characterizes the properties of $\mathrm{PR}$ and its relationship with FRs in human S1. In particular, we investigated the relationship between somatotopic organization and functional interactions of hand representations and reported a 
1 mismatch between the two with respect to palm-finger functional interactions. To

2 further study the link between functional properties of tactile hand representations,

3 physical structure of the hand and natural statistics of tactile stimulation, fMRI mapping

4 data (as in the present study) should be combined with behavioral data (e.g. hand

5 tracking during object manipulation). This would allow investigating inter-individual

6 differences in tactile perception and motor skills and would allow studying brain-body

7 plasticity in clinical conditions like amputation or stroke. 
REFERENCES

Akselrod, M., Martuzzi, R., Serino, A., van der Zwaag, W., Gassert, R., Blanke, O., 2017. Anatomical and functional properties of the foot and leg representation in areas $3 \mathrm{~b}, 1$ and 2 of primary somatosensory cortex in humans: A 7T fMRI study. Neuroimage 159, 473-487. https://doi.org/10.1016/j.neuroimage.2017.06.021

Alexander-Bloch, A.F., Vértes, P.E., Stidd, R., Lalonde, F., Clasen, L., Rapoport, J., Giedd, J., Bullmore, E.T., Gogtay, N., 2013. The anatomical distance of functional connections predicts brain network topology in health and schizophrenia. Cereb. Cortex 23, 127-138. https://doi.org/10.1093/cercor/bhr388

Andersen, K.W., Siebner, H.R., 2018. Mapping dexterity and handedness: recent insights and future challenges. Curr. Opin. Behav. Sci. https://doi.org/10.1016/j.cobeha.2017.12.020

Besle, J., Sánchez-Panchuelo, R.M., Bowtell, R., Francis, S., Schluppeck, D., 2014. Event-related fMRI at $7 \mathrm{~T}$ reveals overlapping cortical representations for adjacent fingertips in S1 of individual subjects. Hum. Brain Mapp. 35, 2027-2043. https://doi.org/10.1002/hbm.22310

Bilecen, D., Seifritz, E., Radu, E.W., Schmid, N., Wetzel, S., Probst, R., Scheffler, K., 2000. Cortical reorganization after acute unilateral hearing loss traced by fMRI. Neurology. https://doi.org/10.1212/WNL.54.3.765

Blankenburg, F., Ruben, J., Meyer, R., Schwiemann, J., Villringer, A., 2003. Evidence for a rostral-tocaudal somatotopic organization in human primary somatosensory cortex with mirror-reversal in areas $3 \mathrm{~b}$ and 1. Cereb. Cortex. https://doi.org/10.1093/cercor/13.9.987

Boakye, M., Huckins, S.C., Szeverenyi, N.M., Taskey, B.I., Hodge, C.J., 2000. Functional magnetic resonance imaging of somatosensory cortex activity produced by electrical stimulation of the median nerve or tactile stimulation of the index finger. J. Neurosurg. 93, 774-783. https://doi.org/10.3171/jns.2000.93.5.0774

Bullock, I.M., Dollar, A.M., 2011. Classifying human manipulation behavior, in: IEEE International Conference on Rehabilitation Robotics. https://doi.org/10.1109/ICORR.2011.5975408

Buonomano, D. V., Merzenich, M.M., 1998. CORTICAL PLASTICITY: From Synapses to Maps. Annu. Rev. Neurosci. 21, 149-186. https://doi.org/10.1146/annurev.neuro.21.1.149

Chino, Y.M., Kaas, J.H., Smith, E.L., Langston, A.L., Cheng, H., 1992. Rapid reorganization of cortical maps in adult cats following restricted deafferentation in retina. Vision Res. https://doi.org/10.1016/0042-6989(92)90021-A

Cooper, M., Seber, G.A.F., 1985. Multivariate Observations. J. Mark. Res. https://doi.org/10.2307/3151376

Di, X., Gohel, S., Kim, E.H., Biswal, B.B., 2013. Task vs. rest-different network configurations between the coactivation and the resting-state brain networks. Front. Hum. Neurosci. https://doi.org/10.3389/fnhum.2013.00493

Ejaz, N., Hamada, M., Diedrichsen, J., 2015. Hand use predicts the structure of representations in sensorimotor cortex. Nat. Neurosci. 103, 1-10. https://doi.org/10.1038/nn.4038

Ercsey-Ravasz, M., Markov, N.T., Lamy, C., VanEssen, D.C., Knoblauch, K., Toroczkai, Z., Kennedy, H., 2013. A Predictive Network Model of Cerebral Cortical Connectivity Based on a Distance Rule. Neuron 80, 184-197. https://doi.org/10.1016/j.neuron.2013.07.036 
bioRxiv preprint doi: https://doi.org/10.1101/2020.09.07.286062; this version posted September 9,2020 . The copyright holder for this preprint (which was not certified by peer review) is the author/funder, who has granted bioRxiv a license to display the preprint in perpetuity. It is made available under aCC-BY-NC 4.0 International license.

Felleman, D.J., Nelson, R.J., Sur, M., Kaas, J.H., 1983. Representations of the body surface in areas 3b and 1 of postcentral parietal cortex of cebus monkeys. Brain Res. 268, 15-26. https://doi.org/10.1016/0006-8993(83)90386-4

Fisher, R. a., Fisher, R. a., 1915. Frequency distribution of the values of the correlation coefficient in samples from an indefinitely large population. Biometrika. https://doi.org/10.2307/2331838

Flor, H. et al, 1995. Phantom-limb pain as a perceptual correlate of cortical reorganization following arm ampFlor, H. et al. (1995). Phantom-limb pain as a perceptual correlate of cortical reorganization following arm amputation. LETTERS TO NATURE.utation. Lett. To Nat. 375, $482-484$.

Glover, G.H., Li, T.Q., Ress, D., 2000. Image-based method for retrospective correction of physiological motion effects in fMRI: RETROICOR. Magn. Reson. Med. 44, 162-167. https://doi.org/10.1002/1522-2594(200007)44:1<162::AID-MRM23>3.0.CO;2-E

Godde, B., Spengler, F., Dinse, H., 1996. Associative pairing of tactile stimulation induces somatosensory cortical reorganization in rats and humans. Neuroreport.

Hashimoto, I., Mashiko, T., Kimura, T., Imada, T., 1999. Are there discrete distal-proximal representations of the index finger and palm in the human somatosensory cortex? A neuromagnetic study. Clin. Neurophysiol. 110, 430-437. https://doi.org/10.1016/S13882457(98)00018-2

Hoijtink, H., Mulder, J., van Lissa, C., Gu, X., 2019. A Tutorial on Testing Hypotheses Using the Bayes Factor. Psychol. Methods. https://doi.org/10.1037/met0000201

Jung, P., Baumgärtner, U., Bauermann, T., Magerl, W., Gawehn, J., Stoeter, P., Treede, R.D., 2003. Asymmetry in the human primary somatosensory cortex and handedness. Neuroimage 19, 913923. https://doi.org/10.1016/S1053-8119(03)00164-2

Jung, P., Baumgärtner, U., Magerl, W., Treede, R.-D., 2008. Hemispheric asymmetry of hand representation in human primary somatosensory cortex and handedness. Clin. Neurophysiol. 119, 2579-86. https://doi.org/10.1016/j.clinph.2008.04.300

Kaas, J.H., Nelson, R.J., Sur, M., Lin, C.S., Merzenich, M.M., 1979. Multiple representations of the body within the primary somatosensory cortex of primates. Science 204, 521-523. https://doi.org/10.1126/science.107591

Kaas, J.H., 1991. Plasticity of sensory and motor maps in adult mammals. Annu. Rev. Neurosci. 14, 137-167. https://doi.org/10.1146/annurev.neuro.14.1.137

Kaas, J.H., 1997. Topographic maps are fundamental to sensory processing. Brain Res. Bull. https://doi.org/10.1016/S0361-9230(97)00094-4

Kaas, J.H., Krubitzer, L.A., Chino, Y.M., Langston, A.L., Polley, E.H., Blair, N., 1990. Reorganization of retinotopic cortical maps in adult mammals after lesions of the retina. Science 248(4952):22931. https://doi.org/10.1126/science.2326637

Kaas, J.H., Merzenich, M.M., Killackey, H.P., 1983. The reorganization of somatosensory cortex following peripheral nerve damage in adult and developing mammals. Annu. Rev. Neurosci. 6, 325-356. https://doi.org/10.1146/annurev.ne.06.030183.001545

Kass, R.E., Raftery, A.E., 1995. Bayes factors. J. Am. Stat. Assoc. https://doi.org/10.1080/01621459.1995.10476572 
bioRxiv preprint doi: https://doi.org/10.1101/2020.09.07.286062; this version posted September 9,2020 . The copyright holder for this preprint (which was not certified by peer review) is the author/funder, who has granted bioRxiv a license to display the preprint in perpetuity. It is made available under aCC-BY-NC 4.0 International license.

Kriegeskorte, N., Mur, M., Bandettini, P., 2008. Representational similarity analysis - connecting the branches of systems neuroscience. Front. Syst. Neurosci. 2, 4. https://doi.org/10.3389/neuro.06.004.2008

Langer, N., Hänggi, J., Müller, N.A., Simmen, H.P., Jäncke, L., 2012. Effects of limb immobilization on brain plasticity. Neurology. https://doi.org/10.1212/WNL.0b013e31823fcd9c

Liepert, J., Tegenthoff, M., Malin, J.P., 1995. Changes of cortical motor area size during immobilization. Electroencephalogr. Clin. Neurophysiol. Electromyogr. https://doi.org/10.1016/0924-980X(95)00194-P

Makin, T.R., Cramer, A.O., Scholz, J., Hahamy, A., Slater, D.H., Tracey, I., Johansen-Berg, H., 2013. Deprivation-related and use-dependent plasticity go hand in hand. Elife 2, 1-15. https://doi.org/10.7554/eLife.01273

Makin, T.R., Flor, H., 2020. Brain (re)organisation following amputation: Implications for phantom limb pain. Neuroimage. https://doi.org/10.1016/j.neuroimage.2020.116943

Marques, J.P., Kober, T., Krueger, G., van der Zwaag, W., Van de Moortele, P.F., Gruetter, R., 2010. MP2RAGE, a self bias-field corrected sequence for improved segmentation and T1-mapping at high field. Neuroimage 49, 1271-1281. https://doi.org/10.1016/j.neuroimage.2009.10.002

Martuzzi, R., van der Zwaag, W., Dieguez, S., Serino, A., Gruetter, R., Blanke, O., 2015. Distinct contributions of Brodmann areas 1 and 2 to body ownership. Soc. Cogn. Affect. Neurosci. 10, 1449-1459. https://doi.org/10.1093/scan/nsv03

Martuzzi, R., van der Zwaag, W., Farthouat, J., Gruetter, R., Blanke, O., 2014. Human finger somatotopy in areas 3b, 1, and 2: A 7T fMRI study using a natural stimulus. Hum. Brain Mapp. 35, 213-226. https://doi.org/10.1002/hbm.22172

Mehring, C., Akselrod, M., Bashford, L., Mace, M., Choi, H., Blüher, M., Buschhoff, A.S., Pistohl, T., Salomon, R., Cheah, A., Blanke, O., Serino, A., Burdet, E., 2019. Augmented manipulation ability in humans with six-fingered hands. Nat. Commun. https://doi.org/10.1038/s41467-01910306-w

Merabet, L.B., Pascual-Leone, A., 2010. Neural reorganization following sensory loss: The opportunity of change. Nat. Rev. Neurosci. https://doi.org/10.1038/nrn2758

Merzenich, M.M., Kaas, J.H., Sur, M., Lin, C.S., 1978. Double representation of the body surface within cytoarchitectonic areas $3 \mathrm{~b}$ and 1 in "SI" in the owl monkey (Aotus trivirgatus). J. Comp. Neurol. 181, 41-73. https://doi.org/10.1002/cne.901810104

Moore, C.I., Stern, C.E., Corkin, S., Fischl, B., Gray, a C., Rosen, B.R., Dale, a M., 2000. Segregation of somatosensory activation in the human rolandic cortex using fMRI. J. Neurophysiol. 84, 558569.

Morgan, V.L., Price, R.R., 2004. The effect of sensorimotor activation on functional connectivity mapping with MRI. Magn. Reson. Imaging. https://doi.org/10.1016/j.mri.2004.07.002

Muret, D., Daligault, S., Dinse, H.R., Delpuech, C., Mattout, J., Reilly, K.T., Farne, A., 2016. Neuromagnetic correlates of adaptive plasticity across the hand-face border in human primary somatosensory cortex. J. Neurophysiol. 2095-2104. https://doi.org/10.1152/jn.00628.2015

Nelson, R.J., Sur, M., Felleman, D.J., Kaas, J.H., 1980. Representations of the body surface in postcentral parietal cortex of Macaca fascicularis. J. Comp. Neurol. 192, 611-43. https://doi.org/10.1002/cne.901920402 
bioRxiv preprint doi: https://doi.org/10.1101/2020.09.07.286062; this version posted September 9,2020 . The copyright holder for this preprint (which was not certified by peer review) is the author/funder, who has granted bioRxiv a license to display the preprint in perpetuity. It is made available under aCC-BY-NC 4.0 International license.

Nili, H., Wingfield, C., Walther, A., Su, L., Marslen-Wilson, W., Kriegeskorte, N., 2014. A Toolbox for Representational Similarity Analysis. PLoS Comput. Biol. 10, e1003553. https://doi.org/10.1371/journal.pcbi.1003553

Oldfield, R.C., 1971. The assessment and analysis of handedness: The Edinburgh inventory. Neuropsychologia 9, 97-113. https://doi.org/10.1016/0028-3932(71)90067-4

Olman, C.A., Van de Moortele, P.F., Schumacher, J.F., Guy, J.R., Uğurbil, K., Yacoub, E., 2010. Retinotopic mapping with spin echo BOLD at 7T. Magn. Reson. Imaging 28, 1258-1269. https://doi.org/10.1016/j.mri.2010.06.001

Pleger, B., Dinse, H.R., Ragert, P., Schwenkreis, P., Malin, J.P., Tegenthoff, M., 2001. Shifts in cortical representations predict human discrimination improvement. Proc. Natl. Acad. Sci. U. S. A. $98,12255-12260$. https://doi.org/10.1073/pnas.191176298

Pleger, B., Foerster, A.F., Ragert, P., Dinse, H.R., Schwenkreis, P., Malin, J.P., Nicolas, V., Tegenthoff, M., 2003. Functional imaging of perceptual learning in human primary and secondary somatosensory cortex. Neuron 40, 643-653. https://doi.org/10.1016/S08966273(03)00677-9

Pont, K., Wallen, M., Bundy, A., 2009. Conceptualising a modified system for classification of in-hand manipulation. Aust. Occup. Ther. J. https://doi.org/10.1111/j.1440-1630.2008.00774.x

Raemaekers, M., Schellekens, W., van Wezel, R.J.A., Petridou, N., Kristo, G., Ramsey, N.F., 2014. Patterns of resting state connectivity in human primary visual cortical areas: A 7T fMRI study. Neuroimage 84, 911-921. https://doi.org/10.1016/j.neuroimage.2013.09.060

Rasmussen, T., Penfield, W., 1947. Further studies of the sensory and motor cerebral cortex of man. Fed. Proc. 6, 452-460.

Saadon-Grosman, N., Tal, Z., Itshayek, E., Amedi, A., Arzy, S., 2015. Discontinuity of cortical gradients reflects sensory impairment. Proc. Natl. Acad. Sci. 112, 16024-16029. https://doi.org/10.1073/pnas.1506214112

Salomon, R., Darulova, J., Narsude, M., Van Der Zwaag, W., 2014. Comparison of an 8-hannel and a 32-channel coil for high-resolution fMRI at 7 T. Brain Topogr. 27, 209-212. https://doi.org/10.1007/s10548-013-0298-6

Salvador, R., Suckling, J., Coleman, M.R., Pickard, J.D., Menon, D., Bullmore, E., 2005. Neurophysiological architecture of functional magnetic resonance images of human brain. Cereb. Cortex 15, 1332-2342. https://doi.org/10.1093/cercor/bhi016

Sanchez-Panchuelo, R.M., Besle, J., Beckett, A., Bowtell, R., Schluppeck, D., Francis, S., 2012. Within-digit functional parcellation of Brodmann areas of the human primary somatosensory cortex using functional magnetic resonance imaging at 7 tesla. J. Neurosci. 32, 15815-15822. https://doi.org/10.1523/jneurosci.2501-12.2012

Sanchez-Panchuelo, R.M., Francis, S., Bowtell, R., Schluppeck, D., 2010. Mapping human somatosensory cortex in individual subjects with 7T functional MRI. J. Neurophysiol. 103, 2544-2556. https://doi.org/10.1152/jn.01017.2009

Sanchez-Panchuelo, R.M., Besle, J., Mougin, O., Gowland, P., Bowtell, R., Schluppeck, D., Francis, S., 2014. Regional structural differences across functionally parcellated Brodmann areas of human primary somatosensory cortex. Neuroimage 93, 221-230. https://doi.org/10.1016/j.neuroimage.2013.03.044 
bioRxiv preprint doi: https://doi.org/10.1101/2020.09.07.286062; this version posted September 9,2020 . The copyright holder for this preprint (which was not certified by peer review) is the author/funder, who has granted bioRxiv a license to display the preprint in perpetuity. It is made available under aCC-BY-NC 4.0 International license.

Sathian, K., Zangaladze, A., 1996. Tactile spatial acuity at the human fingertip and lip: bilateral symmetry and interdigit variability. Neurology 46, 1464-1466. https://doi.org/10.1212/WNL.46.5.1464

Sawilowsky, S.S., 2009. New Effect Size Rules of Thumb. J. Mod. Appl. Stat. Methods. https://doi.org/10.22237/jmasm/1257035100

Schweisfurth, M.A., Frahm, J., Farina, D., Schweizer, R., 2018. Comparison of fMRI digit representations of the dominant and non-dominant hand in the human primary somatosensory cortex. Front. Hum. Neurosci. https://doi.org/10.3389/fnhum.2018.00492

Schweisfurth, M.A., Schweizer, R., Frahm, J., 2011. Functional MRI indicates consistent intra-digit topographic maps in the little but not the index finger within the human primary somatosensory cortex. Neuroimage. https://doi.org/10.1016/j.neuroimage.2011.03.038

Schweisfurth, M. a, Frahm, J., Schweizer, R., 2014. Individual fMRI maps of all phalanges and digit bases of all fingers in human primary somatosensory cortex. Front. Hum. Neurosci. 8, 658. https://doi.org/10.3389/fnhum.2014.00658

Schweizer, R., Voit, D., Frahm, J., 2008. Finger representations in human primary somatosensory cortex as revealed by high-resolution functional MRI of tactile stimulation. Neuroimage 42, 28 35. https://doi.org/10.1016/j.neuroimage.2008.04.184

Serino, A., Akselrod, M., Salomon, R., Martuzzi, R., Blefari, M.L., Canzoneri, E., Rognini, G., Van Der Zwaag, W., Iakova, M., Luthi, F., Amoresano, A., Kuiken, T., Blanke, O., 2017. Upper limb cortical maps in amputees with targeted muscle and sensory reinnervation. Brain 140, 29933011. https://doi.org/10.1093/brain/awx242

Speck, O., Stadler, J., Zaitsev, M., 2008. High resolution single-shot EPI at 7T. Magn. Reson. Mater. Physics, Biol. Med. 21, 73-86. https://doi.org/10.1007/s10334-007-0087-x

Springer, M.S., Meredith, R.W., Gatesy, J., Emerling, C.A., Park, J., Rabosky, D.L., Stadler, T., Steiner, C., Ryder, O.A., Janečka, J.E., Fisher, C.A., Murphy, W.J., 2012. Macroevolutionary Dynamics and Historical Biogeography of Primate Diversification Inferred from a Species Supermatrix. PLoS One. https://doi.org/10.1371/journal.pone.0049521

Stringer, E.A., Chen, L.M., Friedman, R.M., Gatenby, C., Gore, J.C., 2011. Differentiation of somatosensory cortices by high-resolution fMRI at 7T. Neuroimage 54, 1012-1020. https://doi.org/10.1016/j.neuroimage.2010.09.058

Stringer, E.A., Qiao, P.G., Friedman, R.M., Holroyd, L., Newton, A.T., Gore, J.C., Chen, L.M., 2014. Distinct fine-scale fMRI activation patterns of contra- and ipsilateral somatosensory areas $3 \mathrm{~b}$ and 1 in humans. Hum. Brain Mapp. 35, 4841-4857. https://doi.org/10.1002/hbm.22517

Sur, M., Nelson, R.J., Kaas, J.H., 1982. Representations of the body surface in cortical areas $3 \mathrm{~b}$ and 1 of squirrel monkeys: Comparisons with other primates. J. Comp. Neurol. https://doi.org/10.1002/cne.902110207

Syka, J., 2002. Plastic Changes in the Central Auditory System After Hearing Loss, Restoration of Function, and During Learning. Physiol. Rev. https://doi.org/10.1152/physrev.00002.2002

Tourbier S, Aleman-Gomez Y, Griffa A, Bach Cuadra M, Hagmann P (2020). connectomicslab/connectomemapper3: Connectome Mapper v3.0.0-beta-RC2 (Version v3.0.0beta-RC2). Zenodo. http://doi.org/10.5281/zenodo.3475969.

Udin, S.B., Fawcett, J.W., 1988. Formation of Topographic Maps. Annu. Rev. Neurosci. https://doi.org/10.1146/annurev.ne.11.030188.001445 
1 Van Der Zwaag, W., Gruetter, R., Martuzzi, R., 2015. Stroking or buzzing? a comparison of

2 somatosensory touch stimuli using 7 tesla fMRI. PLoS One 10, e0134610.

3 https://doi.org/10.1371/journal.pone.0134610

4 Van Ooyen, A., Carnell, A., De Ridder, S., Tarigan, B., Mansvelder, H.D., Bijma, F., De Gunst, M., Van Pelt, J., 2014. Independently outgrowing neurons and geometry-based synapse formation produce networks with realistic synaptic connectivity. PLoS One. https://doi.org/10.1371/journal.pone.0085858

van Pelt, J., van Ooyen, A., 2013. Estimating neuronal connectivity from axonal and dendritic density fields. Front. Comput. Neurosci. https://doi.org/10.3389/fncom.2013.00160

Whitfield-Gabrieli, S., Nieto-Castanon, A., 2012. Conn: A Functional Connectivity Toolbox for Correlated and Anticorrelated Brain Networks. Brain Connect. 2, 125-141. https://doi.org/10.1089/brain.2012.0073

Zanette, G., Tinazzi, M., Bonato, C., Di Summa, A., Manganotti, P., Polo, A., Fiaschi, A., 1997. Reversible changes of motor cortical outputs following immobilization of the upper limb. Electroencephalogr. Clin. Neurophysiol. - Electromyogr. Mot. Control. https://doi.org/10.1016/S0924-980X(97)00024-6

Zeharia, N., Hertz, U., Flash, T., Amedi, A., 2015. New Whole - Body Sensory - Motor Gradients Revealed Using Phase - Locked Analysis and Verified Using Multivoxel Pattern Analysis and Functional Connectivity. J. Neurosci. 35, 2845-59. https://doi.org/10.1523/JNEUROSCI.424614.2015 


\section{SUPPLEMENTARY FIGURES}

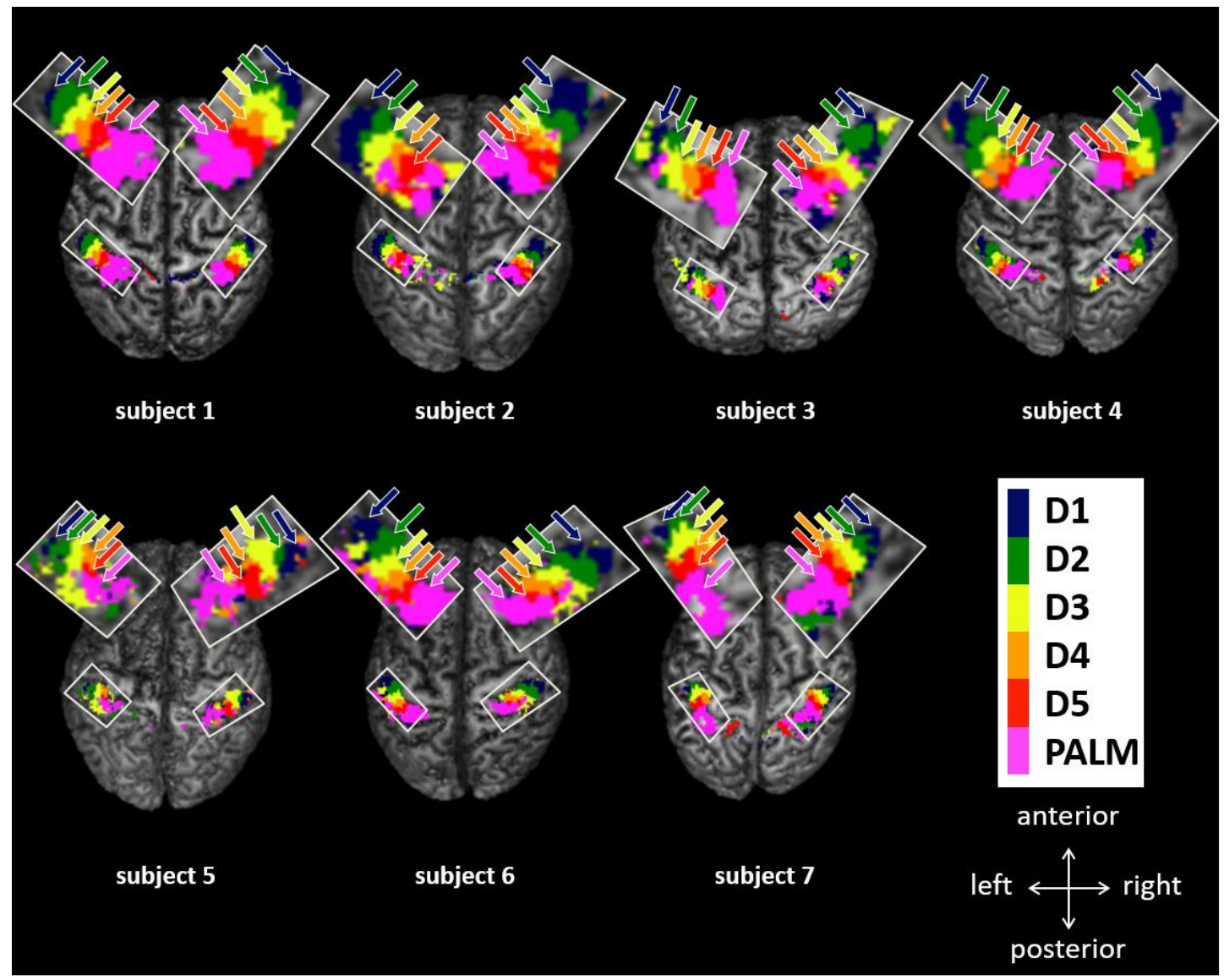

Figure S1. S1 maps from subjects 1-7. S1 hands maps for the right hand in the left hemisphere and the left hand in the right hemisphere are projected and shown from a top view. Note that due to the upward projection used in this visualization, representations located in more superior locations might appear larger as they occlude representations located inferiorly. 
bioRxiv preprint doi: https://doi.org/10.1101/2020.09.07.286062; this version posted September 9, 2020. The copyright holder for this preprint (which was not certified by peer review) is the author/funder, who has granted bioRxiv a license to display the preprint in perpetuity. It is made available under aCC-BY-NC 4.0 International license.

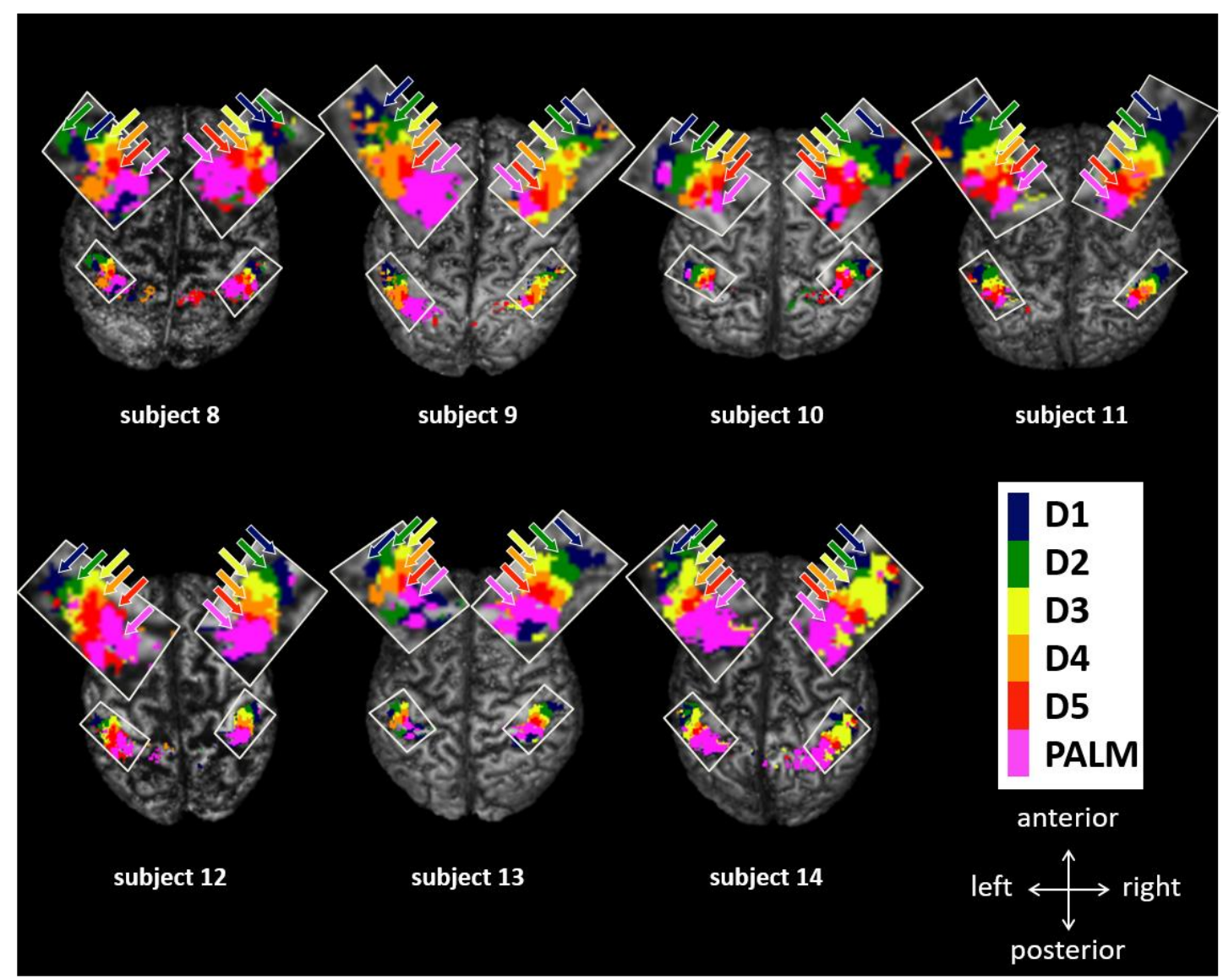

Figure S2. S1 maps from subjects 8-14. S1 hands maps for the right hand in the left hemisphere and the left hand in the right hemisphere are projected and shown from a top view. Note that due to the upward projection used in this visualization, representations located in more superior locations might appear larger as they occlude representations located inferiorly. 

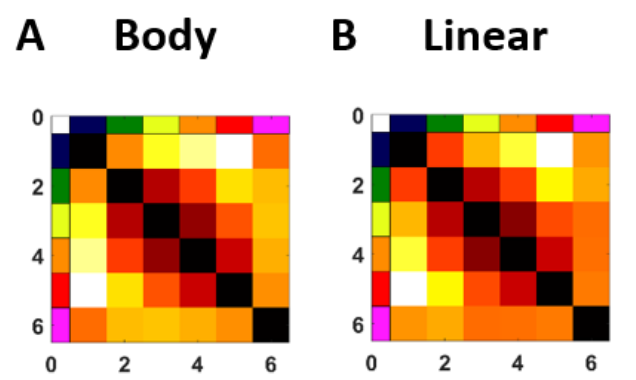

\section{Circular}

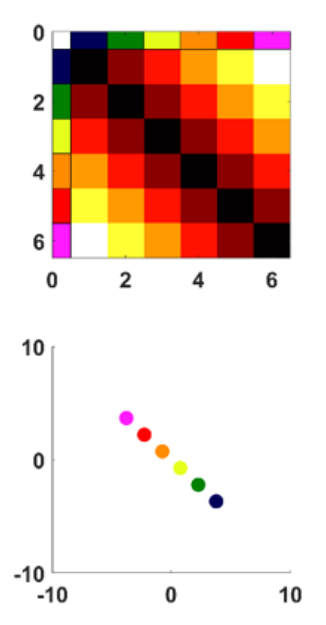

high

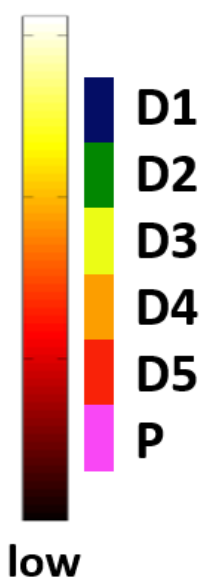

\section{Cort. Dist.}

E Co-activ.
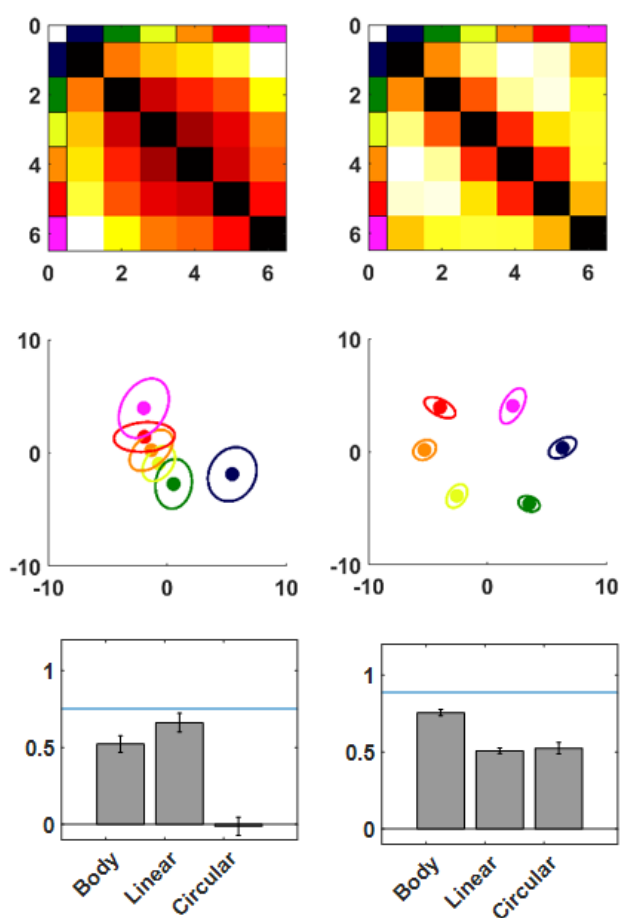
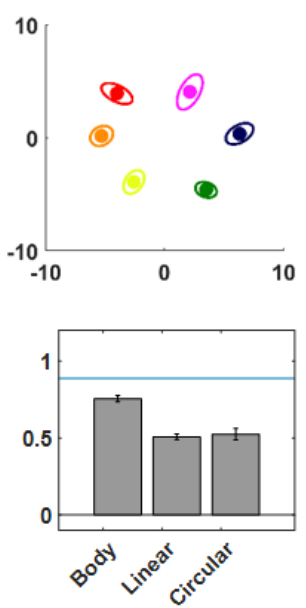

F Multiv. Pat.
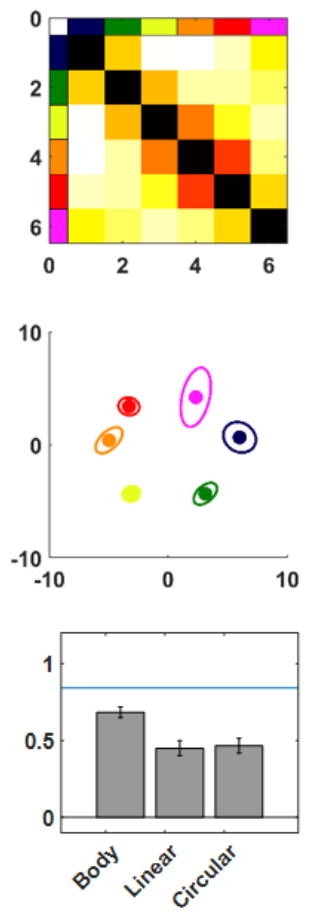

G Func. Conn.
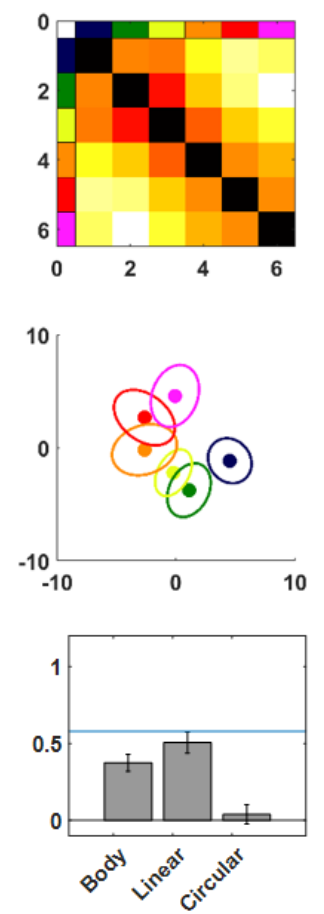

Figure S3. Dissimilarity analysis for the right hand. A-C. Dissimilarity matrix and 2D configuration for the "Body", "Linear" and "Circular" models. D-G. Dissimilarity matrix and 2D configuration for the dissimilarity measures based on cortical distances, co-activations, multi-voxel activity patterns and functional connectivity. The correlations between each dissimilarity measure and the three models are shown in the corresponding bar plots. Asterisks indicate the level of evidence found across Bayesian comparisons. The blue line indicates the noise ceiling. Data presented here are averaged across hands. 


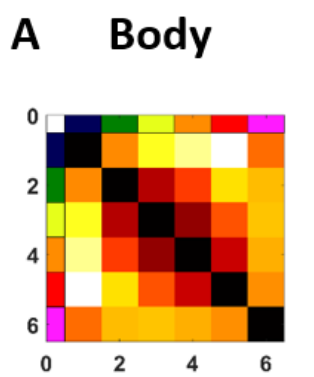

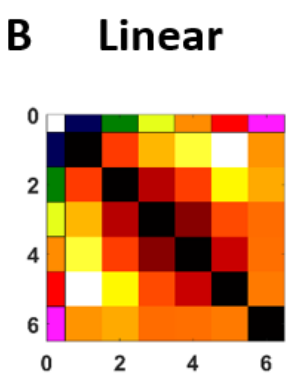

\section{Circular}

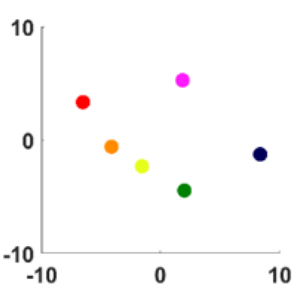

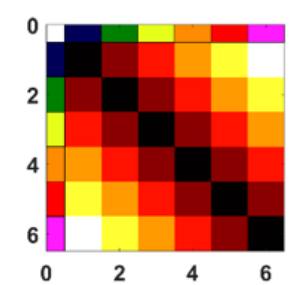

2

high

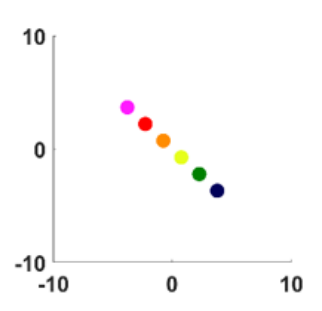

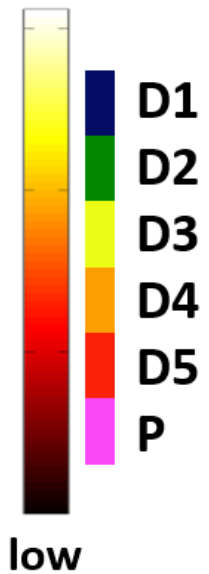

D Cort. Dist.
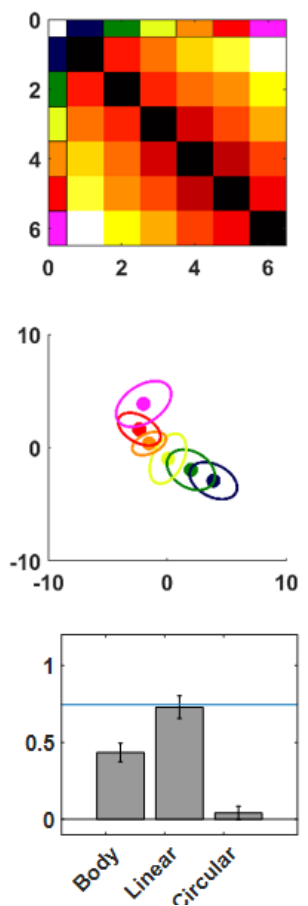

E Co-activ.
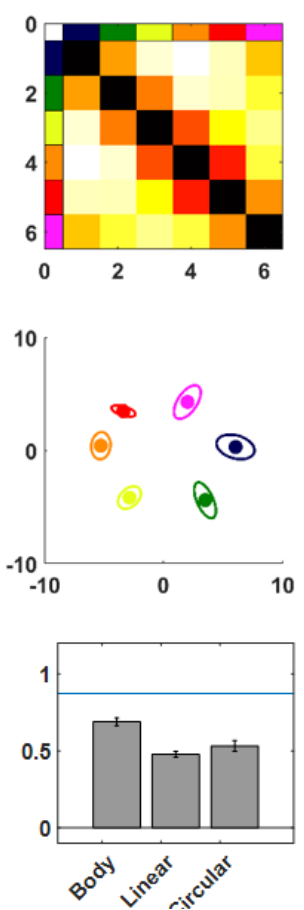

F Multiv. Pat.
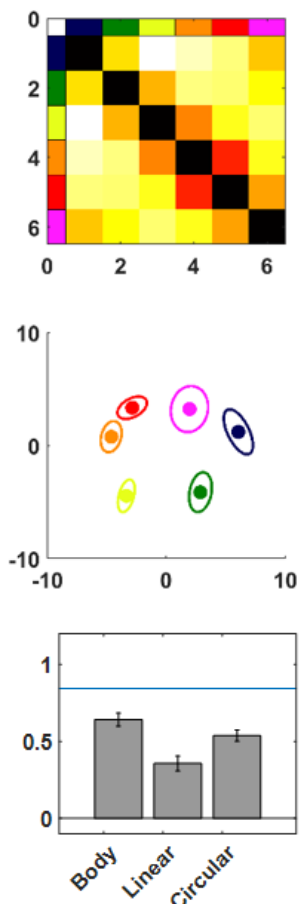

G Func. Conn.
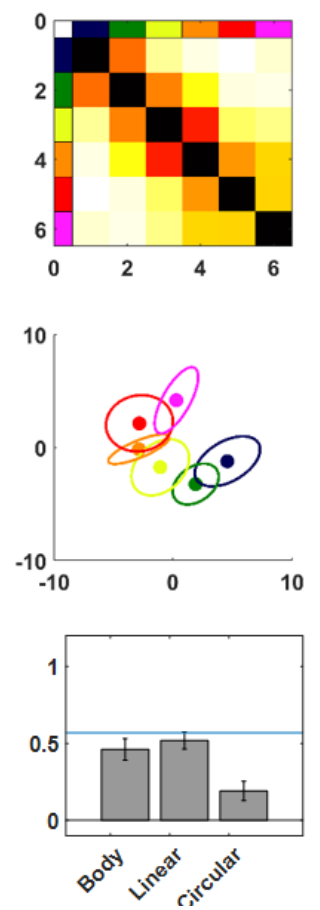

Figure S4. Dissimilarity analysis for the left hand. A-C. Dissimilarity matrix and 2D configuration for the "Body", "Linear" and "Circular" models. D-G. Dissimilarity matrix and 2D configuration for the dissimilarity measures based on cortical distances, co-activations, multi-voxel activity patterns and functional connectivity. The correlations between each dissimilarity measure and the three models are shown in the corresponding bar plots. Asterisks indicate the level of evidence found across Bayesian comparisons. The blue line indicates the noise ceiling. 
A

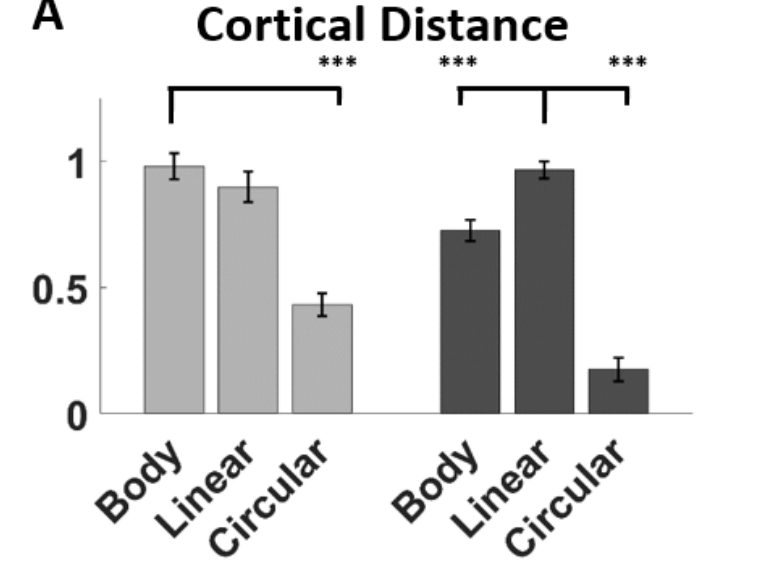

\section{Multivoxel Pattern}

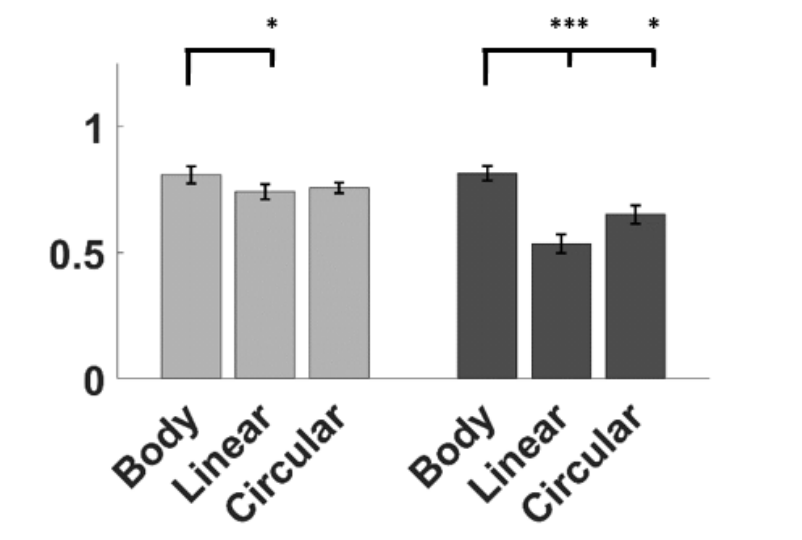

B

\section{Co-activations}

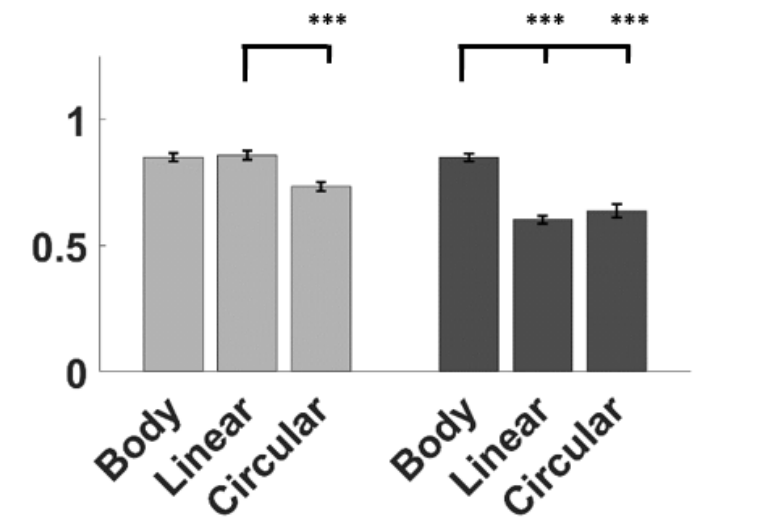

D

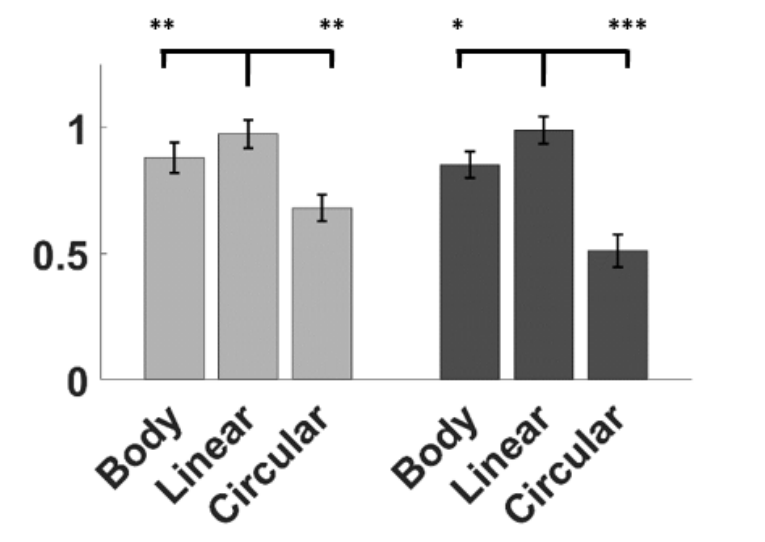

\section{5 fingers}

\section{5 fingers + palm}

$$
\text { bf }>3 * \text {, bf }>10^{* *}, \text { bf }>30 * * *
$$

Figure S5. Comparison between dissimilarity analyses. Dissimilarity analysis was replicated to consider the 5 fingers only by excluding data from the palm. Note that in both cases the variance explained is very high for the best models. However only data including the palm is able to highlight the double dissociation showing that the measures of cortical distance and functional connectivity are better described by the linear model, and that the measures of co-activations and multivoxel activity patterns are better described by the body model. Data were normalized with respect to noise ceiling. 


\begin{tabular}{|c|c|c|}
\hline Measure & Comparison & BF \\
\hline Cortical distance & Body $\neq$ Linear & 173.96 \\
\hline Cortical distance & Body $\neq$ Circular & 55616.24 \\
\hline Cortical distance & Linear $\neq$ Circular & 22670.11 \\
\hline Co-activation & Body $\neq$ Linear & $2.350 \mathrm{e}^{+6}$ \\
\hline Co-activation & Body $\neq$ Circular & 7714.05 \\
\hline Co-activation & Linear $\neq$ Circular & 0.481 \\
\hline Multi-voxel patt. & Body $\neq$ Linear & 16619.11 \\
\hline Multi-voxel patt. & Body $\neq$ Circular & 3.77 \\
\hline Multi-voxel patt. & Linear $\neq$ Circular & 0.90 \\
\hline Functional Conn & Body $\neq$ Linear & 4.67 \\
\hline Functional Conn & Body $\neq$ Circular & 50.23 \\
\hline Functional Conn & Linear $\neq$ Circular & 73.00 \\
\hline
\end{tabular}

Table S1. Bayesian comparisons for dissimilarity analysis with the palm and the five fingers. Separately for each measure of dissimilarity (cortical distances, coactivations, multi-voxel activity patterns and functional connectivity), paired comparisons were computed between each pair of models. Comparisons highlighted in bold are reported in Fig. 6 and Fig.S5. 


\begin{tabular}{|l|l|l|}
\hline \multicolumn{1}{|c|}{ Measure } & \multicolumn{1}{c|}{ Comparison } & \multicolumn{1}{c|}{ BF } \\
\hline Cortical distance & Body $\neq$ Linear & 1.06 \\
\hline Cortical distance & Body $\neq$ Circular & $\mathbf{4 3 5 7 . 3 2}$ \\
\hline Cortical distance & Linear $\neq$ Circular & 247.32 \\
\hline Co-activation & Body $\neq$ Linear & 0.34 \\
\hline Co-activation & Body $\neq$ Circular & 14.86 \\
\hline Co-activation & Linear $\neq$ Circular & $\mathbf{1 8 . 2 2}$ \\
\hline Multi-voxel patt. & Body $\neq$ Linear & $\mathbf{4 . 3 9}$ \\
\hline Multi-voxel patt. & Body $\neq$ Circular & 0.57 \\
\hline Multi-voxel patt. & Linear $\neq$ Circular & 0.27 \\
\hline Functional Conn & Body $\neq$ Linear & $\mathbf{1 4 . 6 7}$ \\
\hline Functional Conn & Body $\neq$ Circular & 2.68 \\
\hline Functional Conn & Linear $\neq$ Circular & $\mathbf{1 7 . 5 8}$ \\
\hline
\end{tabular}

Table S2. Bayesian comparisons for dissimilarity analysis with only the five fingers. Separately for each measure of dissimilarity (cortical distances, co-activations, multi-voxel activity patterns and functional connectivity), paired comparisons were computed between each pair of models. Comparisons highlighted in bold are reported in Fig.S5. 


\section{Right hand}

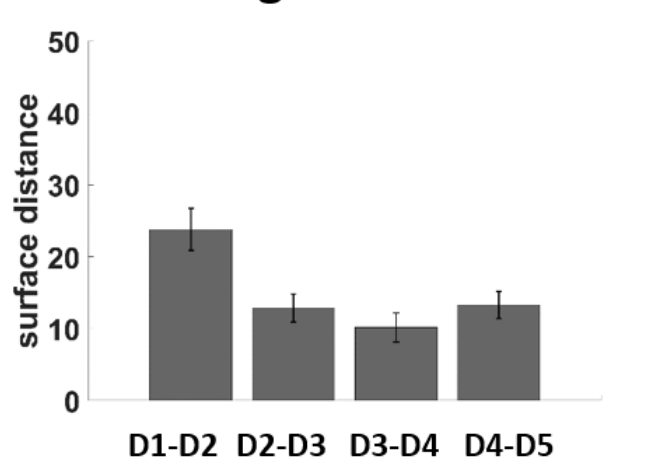

Left hand

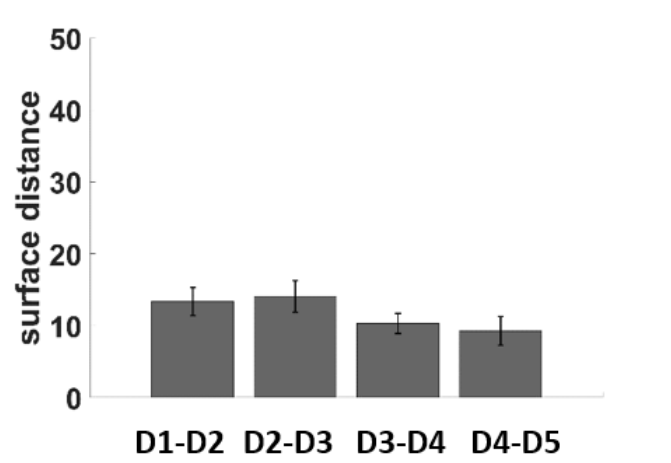

Figure S6. Inter-digit cortical distance. Bar plots of the cortical distances between each pair of FR in the left hemisphere (right hand representations) and in the right hemisphere (left hand representations). Error bars represent the standard error of the mean. A two-way Bayesian ANOVA showed very strong evidence for a main effect of "Finger pair" ( $B F=108.70, P P=0.246)$, no evidence for a main effect of side $(B F=2.32, P P=0.174)$ and positive evidence for an interaction ( $B F=4.36, P P=0.75)$.

Right hand

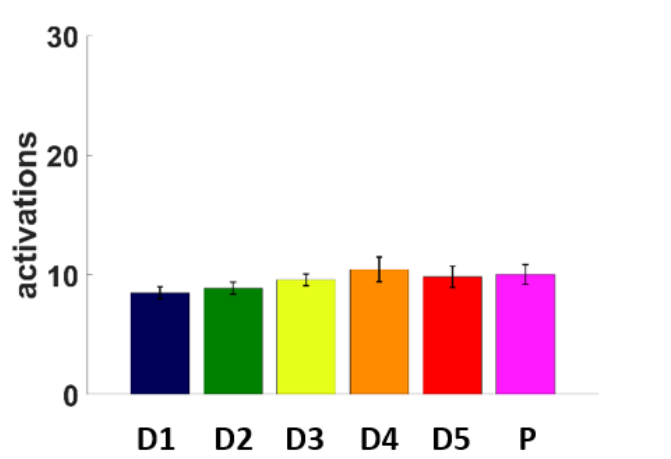

Left hand

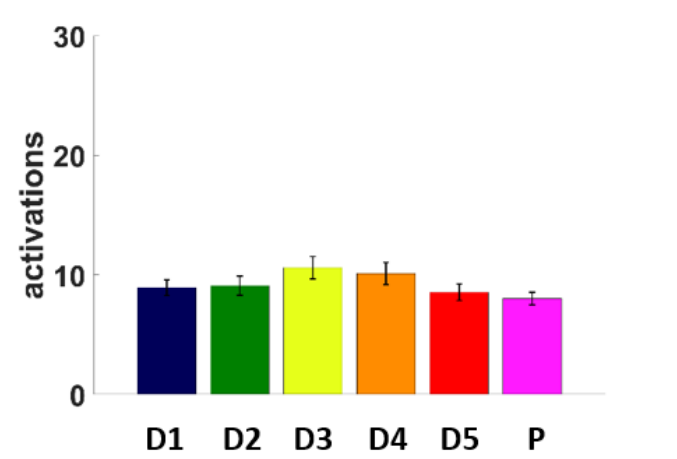

Figure S7. Activations within PR and FRs. Bar plots of beta activations within PR and FRs in the left hemisphere (right hand representations) and in the right hemisphere (left hand representations). Error bars represent the standard error of the mean. A two-way Bayesian ANOVA showed positive evidence for a main effect of "Representation" ( $B F=3.48, P P=0.566)$, no evidence for main effect of side $(B F=0.296, P P=0.166)$ and no evidence for an interaction ( $B F=2.08, P P=0.27)$. 